\title{
Large deviations for locally monotone stochastic partial differential equations driven by Lévy noise
}

\author{
JIE XIONG ${ }^{1}$ and JIANLIANG ZHAI ${ }^{2}$ \\ ${ }^{1}$ Department of Mathematics, Faculty of Science and Technology, University of Macau, Macau, China. \\ E-mail: jiexiong@umac.mo \\ ${ }^{2}$ School of Mathematical Sciences, University of Science and Technology of China, Wu Wen Tsun Key Lab- \\ oratory of Mathematics, Chinese Academy of Science, Hefei, 230026, China.E-mail: zhaijl@ustc.edu.cn
}

We establish a large deviation principle for a type of stochastic partial differential equations (SPDEs) with locally monotone coefficients driven by Lévy noise. The weak convergence method plays an important role.

Keywords: Freidlin-Wentzell type large deviation principle; Levy processes; locally monotone coefficients; stochastic partial differential equations

\section{Introduction}

We shall prove via the weak convergence approach developed in $[5,8,16]$ the Freidlin-Wentzell type large deviation principle (LDP) for a family of locally monotone stochastic partial differentia equations (SPDEs) driven by Lévy processes, these SPDEs include stochastic reactiondiffusion equations, stochastic Burgers type equations, stochastic 2D Navier-Stokes equations and stochastic equations of non-Newtonian fluids.

Let $V$ be a reflexive and separable Banach space, which is densely and continuously injected in a separable Hilbert space $\left(H,\langle\cdot, \cdot\rangle_{H}\right)$. Identifying $H$ with its dual, we get

$$
V \subset H \cong H^{*} \subset V^{*},
$$

where the star '*' denotes the dual spaces. Denote $\langle\cdot, \cdot\rangle_{V^{*}, V}$ the duality between $V^{*}$ and $V$, then we have

$$
\langle u, v\rangle_{V^{*}, V}=\langle u, v\rangle_{H}, \quad \forall u \in H, v \in V .
$$

Fix $T>0$ and let $\left(\Omega, \mathcal{F},\left(\mathcal{F}_{t}\right)_{t \in[0, T]}, \mathbb{P}\right)$ be a complete separable filtration probability space. Let $\mathcal{P}$ be the predictable $\sigma$-field, that is the $\sigma$-field on $[0, T] \times \Omega$ generated by all left continuous and $\mathcal{F}_{t}$-adapted real-valued processes. Further denote by $\mathcal{B F}$ the $\sigma$-field of the progressively measurable sets on $[0, T] \times \Omega$, i.e.

$$
\mathcal{B F}=\left\{O \subset[0, T] \times \Omega: \forall t \in[0, T], O \cap([0, t] \times \Omega) \in \mathcal{B}([0, t]) \otimes \mathcal{F}_{t}\right\},
$$

where $\mathcal{B}([0, t])$ denotes the Borel $\sigma$-field on $[0, t]$. 
Now we consider the following type of SPDEs driven by Lévy processes:

$$
\begin{aligned}
d X_{t}^{\varepsilon} & =\mathcal{A}\left(t, X_{t}^{\varepsilon}\right) d t+\varepsilon \int_{\mathbb{X}} f\left(t, X_{t-}^{\varepsilon}, z\right) \widetilde{N}^{\varepsilon^{-1}}(d t, d z), \\
X_{0}^{\varepsilon} & =x \in H,
\end{aligned}
$$

where $\mathcal{A}:[0, T] \times V \rightarrow V^{*}$ is a $\mathcal{B}([0, T]) \otimes \mathcal{B}(V)$-measurable function. $\mathbb{X}$ is a locally compact Polish space. $N^{\varepsilon^{-1}}$ is a Poisson random measure on $[0, T] \times \mathbb{X}$ with a $\sigma$-finite mean measure $\varepsilon^{-1} \lambda_{T} \otimes \nu, \lambda_{T}$ is the Lebesgue measure on $[0, T]$ and $\nu$ is a $\sigma$-finite measure on $\mathbb{X}$.

$$
\widetilde{N}^{\varepsilon^{-1}}([0, t] \times B)=N^{\varepsilon^{-1}}([0, t] \times B)-\varepsilon^{-1} t v(B), \quad \forall B \in \mathcal{B}(\mathbb{X}) \text { with } v(B)<\infty,
$$

is the compensated Poisson random measure. $f:[0, T] \times V \times \mathbb{X} \rightarrow H$ is a $\mathcal{B}([0, T]) \otimes \mathcal{B}(V) \otimes$ $\mathcal{B}(\mathbb{X})$-measurable function.

The following assumptions are from [4], which guarantee that Eq. (1.1) admits a unique solution. Suppose that there exist constants $\alpha>1, \beta \geq 0, \theta>0, C>0$, positive functions $K$ and $F$ and a function $\rho: V \rightarrow[0,+\infty)$ which is measurable and bounded on the balls, such that the following conditions hold for all $v, v_{1}, v_{2} \in V$ and $t \in[0, T]$ :

(H1) (Hemicontinuity) The map $s \mapsto\left\langle\mathcal{A}\left(t, v_{1}+s v_{2}\right), v\right\rangle_{V^{*}, V}$ is continuous on $\mathbb{R}$.

(H2) (Local monotonicity)

$$
\begin{aligned}
& 2\left\langle\mathcal{A}\left(t, v_{1}\right)-\mathcal{A}\left(t, v_{2}\right), v_{1}-v_{2}\right\rangle_{V^{*}, V}+\int_{\mathbb{X}}\left\|f\left(t, v_{1}, z\right)-f\left(t, v_{2}, z\right)\right\|_{H}^{2} v(d z) \\
& \quad \leq\left(K_{t}+\rho\left(v_{2}\right)\right)\left\|v_{1}-v_{2}\right\|_{H}^{2} .
\end{aligned}
$$

(H3) (Coercivity)

$$
2\langle\mathcal{A}(t, v), v\rangle_{V^{*}, V}+\theta\|v\|_{V}^{\alpha} \leq F_{t}\left(1+\|v\|_{H}^{2}\right) .
$$

(H4) (Growth)

$$
\|\mathcal{A}(t, v)\|_{V^{*}}^{\frac{\alpha}{\alpha-1}} \leq\left(F_{t}+C\|v\|_{V}^{\alpha}\right)\left(1+\|v\|_{H}^{\beta}\right)
$$

Definition 1.1. An $H$-valued cádlág $\mathcal{F}_{t}$-adapted process $\left\{X_{t}^{\varepsilon}\right\}_{t \in[0, T]}$ is called a solution of Eq. (1.1), if for its $d t \times \mathbb{P}$-equivalent class $\widehat{X}^{\varepsilon}$ we have

(1) $\widehat{X}^{\varepsilon} \in L^{\alpha}([0, T] ; V) \cap L^{2}([0, T] ; H), \mathbb{P}$-a.s.;

(2) the following equality holds $\mathbb{P}$-a.s.:

$$
X_{t}^{\varepsilon}=x+\int_{0}^{t} \mathcal{A}\left(s, \bar{X}_{s}^{\varepsilon}\right) d s+\varepsilon \int_{0}^{t} \int_{\mathbb{X}} f\left(s, \bar{X}_{s}^{\varepsilon}, z\right) \widetilde{N}^{\varepsilon^{-1}}(d s, d z), \quad t \in[0, T],
$$

where $\bar{X}^{\varepsilon}$ is any $V$-valued progressively measurable $d t \times \mathbb{P}$ version of $\widehat{X}^{\varepsilon}$. 
Remark 1.1. It is a well-known and typical conclusion in probability theory that $d t \times \mathbb{P}$ equivalent processes/versions are regarded as the same stochastic process, and it is always impossible to find one version to satisfy all required properties. In the above definition, three $d t \times \mathbb{P}$ equivalent versions " $X^{\varepsilon}, \widehat{X}^{\varepsilon}, \bar{X}^{\varepsilon}$ " are implicitly required to ensure that each version satisfies some required properties.

With a minor modification of [4], Theorem 1.2, we have the following existence and uniqueness theorem for the solution of Eq. (1.1).

Theorem 1.1. Suppose that conditions (H1)-(H4) hold for $F, K \in L^{1}\left([0, T] ; \mathbb{R}^{+}\right)$, and there exists a constant $\gamma<\frac{\theta}{2 \beta}$ and $G \in L^{1}\left([0, T] ; \mathbb{R}^{+}\right)$such that for all $t \in[0, T]$ and $v \in V$ we have

$$
\begin{aligned}
\int_{\mathbb{X}}\|f(t, v, z)\|_{H}^{2} v(d z) & \leq F_{t}\left(1+\|v\|_{H}^{2}\right)+\gamma\|v\|_{V}^{\alpha} ; \\
\int_{\mathbb{X}}\|f(t, v, z)\|_{H}^{\beta+2} v(d z) & \leq G_{t}\left(1+\|v\|_{H}^{\beta+2}\right) ; \\
\rho(v) & \leq C\left(1+\|v\|_{V}^{\alpha}\right)\left(1+\|v\|_{H}^{\beta}\right) .
\end{aligned}
$$

Then

(1) For any $x \in L^{\beta+2}\left(\Omega, \mathcal{F}_{0}, \mathbb{P} ; H\right)$, (1.1) has a unique solution $\left\{X_{t}^{\varepsilon}\right\}_{t \in[0, T]}$.

(2) If $\gamma$ is small enough, then

$$
\begin{gathered}
\mathbb{E}\left(\sup _{t \in[0, T]}\left\|X_{t}^{\varepsilon}\right\|_{H}^{\beta+2}\right)+\mathbb{E} \int_{0}^{T}\left\|X_{t}^{\varepsilon}\right\|_{H}^{\beta}\left\|X_{t}^{\varepsilon}\right\|_{V}^{\alpha} d t \\
\leq C_{\varepsilon}\left(\mathbb{E}\|x\|_{H}^{\beta+2}+\int_{0}^{T} G_{t} d t+\int_{0}^{T} F_{t} d t\right) .
\end{gathered}
$$

Remark 1.2. The assumptions in Theorem 1.1 are satisfied by a very large class of SPDEs driven by a multiplicative pure jump Lévy noise, including the stochastic porous medium equation, stochastic p-Laplace equation, stochastic Burgers type equations, stochastic 2D Navier-Stokes equations and many other stochastic hydrodynamical systems. Section 2 in [4] presents many concrete examples to illustrate the applications of this theorem. It is omitted in this paper.

Our aim in the present paper is to establish a LDP for the solution of (1.1) as $\varepsilon \rightarrow 0$ on $D([0, T], H)$, the space of $H$-valued càdlàg functions on $[0, T]$.

In the past three decades, there are numerous literatures about the LDP for stochastic evolution equations (SEEs) and SPDEs driven by Gaussian processes (cf. [3,6,7,9-12,15,19,20,22,23,25, $26,31,32]$, etc.). Many of these results were obtained by using the weak convergence approach for the case of Gaussian noise, introduced in [6,7], see, for example, [3,6,7,15,20,22,23,25,32]. This approach has been proved to be very effective for various finite/infinite-dimensional stochastic dynamical systems. One of the main advantages of this approach is that one only needs to make some necessary moment estimates. 
The situations for SEEs and SPDEs driven by Lévy noise are drastically different because of the appearance of the jumps. There are only a few results on this topic so far. The first paper on LDP for SEEs of jump type is [24] where the additive noise is considered. The study of LDP for multiplicative Lévy noise has been carried out as well, for example, [27] and [5] for SEEs where the LDP was established on a space larger (hence, with a weaker topology) than the actual state space of the solution, [29] for SEEs on the actual state space, [30] for the 2-D stochastic NavierStokes equations (SNSEs). Before [30], [28] dealt with the 2-D SNSEs driven by additive Lévy noise. We also refer to $[2,13,14,17]$ for related results.

To obtain our result, we will use the weak convergence approach introduced in $[5,8]$ for the case of Poisson random measures. This approach is a powerful tool to prove the LDP for SEEs and SPDEs driven by Lévy noise, which has been applied for several dynamical systems. The weak convergence method was first used in [5] to obtain LDP for SPDEs on co-nuclear spaces driven by Lévy noises, and then in [29] for SPDEs on Hilbert spaces with regular coefficients. Paper [30] deals with the 2-D SNSEs driven by multiplicative Lévy noise. Bao and Yuan [2] established a LDP for a class of stochastic functional differential equations of neutral type driven by a finite-dimensional Wiener process and a stationary Poisson random measure.

Monotone method is a main tool to prove the existence and uniqueness of SPDEs, and it can tackle a large class of SPDEs, for more details, see [4,21] and references therein. Working in the framework of [4], the purpose of this paper is to establish a LDP for a family of locally monotone SPDEs (1.1) driven by pure jumps. In addition to the difficulties caused by the jumps, much of our problem is to deal with the monotone operator $\mathcal{A}$.

This paper is organized as follows. In Section 2, we will recall the abstract criteria for LDP obtained in [8]. In Section 3, we will show the main result of this paper. Section 4 and Section 5 is devoted to prove prior results on the controlled SPDEs (4.4), which play a key role in this paper. The entire Section 6 is to establish the LDP for (1.1).

\section{Preliminaries}

\subsection{Poisson random measure}

For convenience of the reader, we shall adopt the notation in [5] and [8]. Recall that $\mathbb{X}$ is a locally compact Polish space. Denote by $\mathcal{M}_{\mathrm{FC}}(\mathbb{X})$ the collection of all measures on $(\mathbb{X}, \mathcal{B}(\mathbb{X}))$ such that $v(K)<\infty$ for any compact $K \in \mathcal{B}(\mathbb{X})$. Denote by $C_{c}(\mathbb{X})$ the space of continuous functions with compact supports, endow $\mathcal{M}_{\mathrm{FC}}(\mathbb{X})$ with the weakest topology such that for every $f \in C_{c}(\mathbb{X})$, the function

$$
v \rightarrow\langle f, v\rangle=\int_{\mathbb{X}} f(u) d v(u),
$$

is continuous for $v \in \mathcal{M}_{\mathrm{FC}}(\mathbb{X})$. This topology can be metrized such that $\mathcal{M}_{\mathrm{FC}}(\mathbb{X})$ is a Polish space (see e.g. [8]).

Fixing $T \in(0, \infty)$, we denote $\mathbb{X}_{T}=[0, T] \times \mathbb{X}$ and $v_{T}=\lambda_{T} \otimes v$ with $\lambda_{T}$ being Lebesgue measure on $[0, T]$ and $v \in \mathcal{M}_{\mathrm{FC}}(\mathbb{X})$. Let $\mathbf{n}$ be a Poisson random measure on $\mathbb{X}_{T}$ with intensity measure $v_{T}$, it is well-known [18] that $\mathbf{n}$ is an $\mathcal{M}_{\mathrm{FC}}\left(\mathbb{X}_{T}\right)$ valued random variable such that 
(i) for each $B \in \mathcal{B}\left(\mathbb{X}_{T}\right)$ with $v_{T}(B)<\infty, \mathbf{n}(B)$ is Poisson distributed with mean $v_{T}(B)$;

(ii) for disjoint $B_{1}, \ldots, B_{k} \in \mathcal{B}\left(\mathbb{X}_{T}\right), \mathbf{n}\left(B_{1}\right), \ldots, \mathbf{n}\left(B_{k}\right)$ are mutually independent random variables.

For notational simplicity, we write from now on

$$
\mathbb{M}=\mathcal{M}_{\mathrm{FC}}\left(\mathbb{X}_{T}\right)
$$

and denote by $\mathbb{P}$ the probability measure induced by $\mathbf{n}$ on $(\mathbb{M}, \mathcal{B}(\mathbb{M}))$. Under $\mathbb{P}$, the canonical map, $N: \mathbb{M} \rightarrow \mathbb{M}, N(m) \doteq m$, is a Poisson random measure with intensity measure $v_{T}$. With applications to large deviations in mind, we also consider, for $\theta>0$, probability measures $\mathbb{P}_{\theta}$ on $(\mathbb{M}, \mathcal{B}(\mathbb{M}))$ under which $N$ is a Poisson random measure with intensity $\theta v_{T}$. The corresponding expectation operators will be denoted by $\mathbb{E}$ and $\mathbb{E}_{\theta}$, respectively.

For further use, simply denote

$$
\mathbb{Y}=\mathbb{X} \times[0, \infty), \quad \mathbb{Y}_{T}=[0, T] \times \mathbb{Y}, \quad \overline{\mathbb{M}}=\mathcal{M}_{\mathrm{FC}}\left(\mathbb{Y}_{T}\right)
$$

Let $\overline{\mathbb{P}}$ be the unique probability measure on $(\overline{\mathbb{M}}, \mathcal{B}(\overline{\mathbb{M}}))$ under which the canonical map, $\bar{N}$ : $\overline{\mathrm{M}} \rightarrow \overline{\mathrm{M}}, \bar{N}(\bar{m}) \doteq \bar{m}$, is a Poisson random measure with intensity measure $\bar{v}_{T}=\lambda_{T} \otimes v \otimes \lambda_{\infty}$, with $\lambda_{\infty}$ being Lebesgue measure on $[0, \infty)$. The corresponding expectation operator will be denoted by $\overline{\mathbb{E}}$. Let $\mathcal{F}_{t} \doteq \sigma\{\bar{N}((0, s] \times A): 0 \leq s \leq t, A \in \mathcal{B}(\mathbb{Y})\}$, and let $\overline{\mathcal{F}}_{t}$ denote the completion under $\overline{\mathbb{P}}$. We denote by $\overline{\mathcal{P}}$ the predictable $\sigma$-field on $[0, T] \times \overline{\mathbb{M}}$ with the filtration $\left\{\overline{\mathcal{F}}_{t}: 0 \leq t \leq T\right\}$ on $(\overline{\mathbb{M}}, \mathcal{B}(\overline{\mathbb{M}}))$. Let $\overline{\mathbb{A}}$ be the class of all $(\overline{\mathcal{P}} \otimes \mathcal{B}(\mathbb{X})) / \mathcal{B}([0, \infty))$-measurable maps $\varphi: \mathbb{X}_{T} \times \overline{\bar{M}} \rightarrow$ $[0, \infty)$. For $\varphi \in \overline{\mathbb{A}}$, we shall suppress the argument $\bar{m}$ in $\varphi(s, x, \bar{m})$ and simply write $\varphi(s, x)=$ $\varphi(s, x, \bar{m})$. Define a counting process $N^{\varphi}$ on $\mathbb{X}_{T}$ by

$$
N^{\varphi}((0, t] \times U)=\int_{(0, t] \times U} \int_{(0, \infty)} 1_{[0, \varphi(s, x)]}(r) \bar{N}(d s d x d r), \quad t \in[0, T], U \in \mathcal{B}(\mathbb{X}) .
$$

The above $N^{\varphi}$ is called a controlled random measure, with $\varphi$ selecting the intensity for the points at location $x$ and time $s$, in a possibly random but non-anticipating way. When $\varphi(s, x, \bar{m}) \equiv \theta \in(0, \infty)$, we write $N^{\varphi}=N^{\theta}$. Note that $N^{\theta}$ has the same distribution with respect to $\overline{\mathbb{P}}$ as $N$ has with respect to $\mathbb{P}_{\theta}$. The following is a representation formula proved in [8].

Theorem 2.1. Let $F \in M_{b}(\mathbb{M})$. Then, for $\theta>0$,

$$
-\log \mathbb{E}_{\theta}\left(e^{-F(N)}\right)=-\log \overline{\mathbb{E}}\left(e^{-F\left(N^{\theta}\right)}\right)=\inf _{\varphi \in \overline{\mathcal{A}}} \overline{\mathbb{E}}\left[\theta L_{T}(\varphi)+F\left(N^{\theta \varphi}\right)\right] .
$$

\subsection{A general criterion for large deviation principle ([5], Theorem 2.4)}

We first state the large deviation principle we are concerned with. Let $\left\{X^{\varepsilon}, \varepsilon>0\right\} \equiv\left\{X^{\varepsilon}\right\}$ be a family of random variables defined on a probability space $(\Omega, \mathcal{F}, \mathbb{P})$ and taking values in a Polish space $\mathcal{E}$. Denote the expectation with respect to $\mathbb{P}$ by $\mathbb{E}$. The theory of large deviations is concerned with events $A$ for which probability $\mathbb{P}\left(X^{\varepsilon} \in A\right)$ converges to zero exponentially fast as $\varepsilon \rightarrow 0$. The exponential decay rate of such probabilities is typically expressed in terms of a 'rate function' $I$ defined as below. 
Definition 2.1 (Rate function). A function $I: \mathcal{E} \rightarrow[0, \infty]$ is called a rate function on $\mathcal{E}$, if for each $M<\infty$ the level set $\{y \in \mathcal{E}: I(y) \leq M\}$ is a compact subset of $\mathcal{E}$. For $A \in \mathcal{B}(\mathcal{E})$, we define $I(A) \doteq \inf _{y \in A} I(y)$.

Definition 2.2 (Large deviation principle). Let $I$ be a rate function on $\mathcal{E}$. The sequence $\left\{X^{\varepsilon}\right\}$ is said to satisfy a large deviation principle (LDP) on $\mathcal{E}$ with rate function $I$ if the following two conditions hold.

(a) LDP upper bound. For each closed subset $F$ of $\mathcal{E}$,

$$
\limsup _{\varepsilon \rightarrow 0} \varepsilon \log \mathbb{P}\left(X^{\varepsilon} \in F\right) \leq-I(F) .
$$

(b) LDP lower bound. For each open subset $G$ of $\mathcal{E}$,

$$
\liminf _{\varepsilon \rightarrow 0} \varepsilon \log \mathbb{P}\left(X^{\varepsilon} \in G\right) \geq-I(G) .
$$

Next, we recall the general criterion for large deviation principles established in [5]. Let $\left\{\mathcal{G}^{\varepsilon}\right\}_{\varepsilon>0}$ be a family of measurable maps from $\mathbb{M}$ to $\mathbb{U}$, where $\mathbb{M}$ is introduced in (2.1) and $\mathbb{U}$ is a Polish space. We present below a sufficient condition for LDP of the family $Z^{\varepsilon}=\mathcal{G}^{\varepsilon}\left(\varepsilon N^{\varepsilon^{-1}}\right)$, as $\varepsilon \rightarrow 0$.

Define

$$
S^{N}=\left\{g: \mathbb{X}_{T} \rightarrow[0, \infty): L_{T}(g) \leq N\right\},
$$

a function $g \in S^{N}$ can be identified with a measure $\nu_{T}^{g} \in \mathbb{M}$, defined by

$$
v_{T}^{g}(A)=\int_{A} g(s, x) v_{T}(d s d x), \quad A \in \mathcal{B}\left(\mathbb{X}_{T}\right) .
$$

This identification induces a topology on $S^{N}$ under which $S^{N}$ is a compact space, see the Appendix of [5]. Throughout this paper, we use this topology on $S^{N}$. Denote $S=\bigcup_{N=1}^{\infty} S^{N}$ and $\overline{\mathbb{A}}^{N}:=\left\{\varphi \in \overline{\mathbb{A}}\right.$ and $\varphi(\omega) \in S^{N}, \overline{\mathbb{P}}$-a.s. $\}$.

Let $\left\{K_{n} \subset \mathbb{X}, n=1,2, \ldots\right\}$ be an increasing sequence of compact sets such that $\bigcup_{n=1}^{\infty} K_{n}=\mathbb{X}$. For each $n$, let

$$
\begin{aligned}
\overline{\mathbb{A}}_{b, n}= & \left\{\varphi \in \overline{\mathbb{A}}: \text { for all }(t, \omega) \in[0, T] \times \overline{\mathbb{M}}, n \geq \varphi(t, x, \omega) \geq 1 / n \text { if } x \in K_{n}\right. \\
& \text { and } \left.\varphi(t, x, \omega)=1 \text { if } x \in K_{n}^{c}\right\},
\end{aligned}
$$

and let $\overline{\mathbb{A}}_{b}=\bigcup_{n=1}^{\infty} \overline{\mathbb{A}}_{b, n}$. Define $\tilde{\mathbb{A}}^{N}=\overline{\mathbb{A}}^{N} \cap\left\{\phi: \phi \in \overline{\mathbb{A}}_{b}\right\}$.

Condition 2.1. There exists a measurable map $\mathcal{G}^{0}: \mathbb{M} \rightarrow \mathbb{U}$ such that the following hold.

(a) For all $N \in \mathbb{N}$, let $g_{n}, g \in S^{N}$ be such that $g_{n} \rightarrow g$ as $n \rightarrow \infty$. Then

$$
\mathcal{G}^{0}\left(v_{T}^{g_{n}}\right) \rightarrow \mathcal{G}^{0}\left(v_{T}^{g}\right) \quad \text { in } \mathbb{U} .
$$


(b) For all $N \in \mathbb{N}$, let $\varphi_{\varepsilon}, \varphi \in \tilde{\mathbb{A}}^{N}$ be such that $\varphi_{\varepsilon}$ converges in distribution to $\varphi$ as $\varepsilon \rightarrow 0$. Then

$$
\mathcal{G}^{\varepsilon}\left(\varepsilon N^{\varepsilon^{-1} \varphi_{\varepsilon}}\right) \Rightarrow \mathcal{G}^{0}\left(v_{T}^{\varphi}\right)
$$

In this paper, we use the symbol " $\Rightarrow$ " to denote convergence in distribution.

For $\phi \in \mathbb{U}$, define $\mathbb{S}_{\phi}=\left\{g \in S: \phi=\mathcal{G}^{0}\left(\nu_{T}^{g}\right)\right\}$. Let $I: \mathbb{U} \rightarrow[0, \infty]$ be defined by

$$
I(\phi)=\inf _{g \in \mathbb{S}_{\phi}} L_{T}(g), \quad \phi \in \mathbb{U} .
$$

By convention, $I(\phi)=\infty$ if $\mathbb{S}_{\phi}=\varnothing$.

The following criterion for LDP was established in Theorem 2.4 of [5], which is a strengthened form of Theorem 4.2 of [8], and for applications, it is more useful.

Theorem 2.2. For $\varepsilon>0$, let $Z^{\varepsilon}$ be defined by $Z^{\varepsilon}=\mathcal{G}^{\varepsilon}\left(\varepsilon N^{\varepsilon^{-1}}\right)$, and suppose that Condition 2.1 holds. Then the family $\left\{Z^{\varepsilon}\right\}_{\varepsilon>0}$ satisfies a large deviation principle with the rate function I defined by (2.5).

\section{LDP for Eq. (1.1)}

Assume that $X_{0}=x \in H$ is deterministic. Let $X^{\varepsilon}$ be the $H$-valued solution to Eq. (1.1) with initial value $x$. In this section, we state the LDP on $D([0, T], H)$ for $\left\{X^{\varepsilon}\right\}$ under suitable assumptions.

Take $\mathbb{U}=D([0, T], H)$ in Condition 2.1 with the Skorokhod topology $\mathbb{U}_{S}$. We know that $\left(\mathbb{U}, \mathbb{U}_{S}\right)$ is a Polish space. For $p>0$, define

$$
\begin{aligned}
\mathcal{H}_{p}= & \left\{h:[0, T] \times \mathbb{X} \rightarrow \mathbb{R}^{+}: \exists \delta>0, \text { s.t. } \forall \Gamma \in \mathcal{B}([0, T]) \otimes \mathcal{B}(\mathbb{X})\right. \\
& \text { with } \left.v_{T}(\Gamma)<\infty, \text { we have } \int_{\Gamma} \exp \left(\delta h^{p}(t, y)\right) v(d y) d t<\infty\right\} .
\end{aligned}
$$

Remark 3.1. It is easy to check that $\mathcal{H}_{p} \subset \mathcal{H}_{p^{\prime}}$ for any $p^{\prime} \in(0, p)$ and

$$
\left\{h:[0, T] \times \mathbb{X} \rightarrow \mathbb{R}^{+}, \sup _{(t, y) \in[0, T] \times \mathbb{X}} h(t, y)<\infty\right\} \subset \mathcal{H}_{p}, \quad \forall p>0 .
$$

To study LDP of Eq. (1.1), besides the assumptions (H1)-(H4), we further need

(H5) There exist $\eta_{0}>0, p \geq \Upsilon$ with $\Upsilon:=\frac{2 \beta(\alpha-1)\left(\alpha+\eta_{0}\right)}{\alpha} \vee \frac{4(\alpha-1)\left(\alpha+\eta_{0}\right)}{\alpha} \vee 4 \vee(\beta+2)$, and $L_{f} \in L_{2}\left(v_{T}\right) \cap L_{4}\left(v_{T}\right) \cap L_{\beta+2}\left(v_{T}\right) \cap L_{\Upsilon}\left(v_{T}\right) \cap L_{\frac{\Upsilon}{2}}\left(v_{T}\right) \cap \mathcal{H}_{p}$ such that

$$
\|f(t, v, z)\|_{H} \leq L_{f}(t, z)\left(1+\|v\|_{H}\right), \quad \forall(t, v, z) \in[0, T] \times V \times \mathbb{X} .
$$


(H6) There exists $G_{f} \in L_{2}\left(v_{T}\right) \cap \mathcal{H}_{2}$ such that

$$
\left\|f\left(t, v_{1}, z\right)-f\left(t, v_{2}, z\right)\right\|_{H} \leq G_{f}(t, z)\left\|v_{1}-v_{2}\right\|_{H}, \quad \forall(t, z) \in[0, T] \times \mathbb{X}, v_{1}, v_{2} \in V
$$

Remark 3.2. It is easy to check that

$$
\begin{aligned}
& L_{2}\left(v_{T}\right) \cap\left\{h:[0, T] \times \mathbb{X} \rightarrow \mathbb{R}^{+},\|h\|_{\infty}<\infty\right\} \\
& \quad \subset L_{2}\left(v_{T}\right) \cap L_{4}\left(v_{T}\right) \cap L_{\beta+2}\left(v_{T}\right) \cap L_{\Upsilon}\left(v_{T}\right) \cap L_{\frac{\Upsilon}{2}}\left(v_{T}\right) \cap \mathcal{H}_{p},
\end{aligned}
$$

where $\|h\|_{\infty}=\sup _{(t, y) \in[0, T] \times \mathbb{X}} h(t, y)$.

It follows from Theorem 1.1 that, for every $\varepsilon>0$, there exists a measurable map $\mathcal{G}^{\varepsilon}: \overline{\mathbb{M}} \rightarrow$ $D([0, T] ; H)$ such that, for any Poisson random measure $\mathbf{n}^{\varepsilon^{-1}}$ on $[0, T] \times \mathbb{X}$ with mean measure $\varepsilon^{-1} \lambda_{T} \otimes \nu$ given on some probability space, $\mathcal{G}^{\varepsilon}\left(\varepsilon \mathbf{n}^{\varepsilon^{-1}}\right)$ is the unique solution $X^{\varepsilon}$ of (1.1) with $\widetilde{N}^{\varepsilon^{-1}}$ replaced by $\widetilde{\mathbf{n}}^{\varepsilon^{-1}}$, here $\widetilde{\mathbf{n}}^{\varepsilon^{-1}}$ is the compensated Poisson random measure of $\mathbf{n}^{\varepsilon^{-1}}$.

To state our main result, we need to introduce the map $\mathcal{G}^{0}$. Recall $S$ given in Section 2.2. For $g \in S$, consider the following deterministic PDE (the skeleton equation):

$$
X_{t}^{0, g}=x+\int_{0}^{t} \mathcal{A}\left(s, X_{s}^{0, g}\right) d s+\int_{0}^{t} f\left(s, X_{s}^{0, g}, z\right)(g(s, z)-1) v(d z) d s, \quad \text { in } V^{*} .
$$

By Proposition 5.1 below, this equation has a unique solution $X^{0, g} \in C([0, T], H) \cap$ $L^{\alpha}([0, T], V)$. Define

$$
\mathcal{G}^{0}\left(v_{T}^{g}\right):=X^{0, g}, \quad \forall g \in S .
$$

Let $I: \mathbb{U}=D([0, T], H) \rightarrow[0, \infty]$ be defined as in (2.5). The following is the main result of this paper.

Theorem 3.1. Assume that (H1)-(H6) and (1.4) hold. Then the family $\left\{X^{\varepsilon}\right\}_{\varepsilon>0}$ satisfies an LDP on $D([0, T], H)$ with the rate function I under the topology of uniform convergence.

Proof. According to Theorem 2.2, we only need to verify Condition 2.1, which will be done in the last section.

\section{Tightness of $\mathcal{G}^{\varepsilon}\left(\varepsilon N^{\varepsilon^{-1}} \varphi_{\varepsilon}\right)$}

The main result of this section is Proposition 4.1 in which we prove the tightness of the solutions $\widetilde{X}^{\varepsilon}$ of the controlled SPDEs (4.4). To this end, $\widetilde{X}^{\varepsilon}$ is written as the summation of three terms $Y^{\varepsilon}+Z^{\varepsilon}+M^{\varepsilon}$, and their tightness are proved separately. In fact, the martingale part $M^{\varepsilon}$ is proved to converge to 0 by an estimate of the $H$-norm of $\widetilde{X}^{\varepsilon}$ which is given in Lemma 4.4. The other two parts $Z^{\varepsilon}$ and $Y^{\varepsilon}$ are of finite variations, and their tightness follows from Ascoli-Arzelá's theorem and from Kolmogorov's criterion respectively. The keys in applying these theorems are again in obtaining good estimates of the norms of $\widetilde{X}^{\varepsilon}$. For $Z^{\varepsilon}$, the $H$-norm is again needed. 
However, for $Y^{\varepsilon}$, the $V$-norm is needed which is derived in Lemma 4.5. As auxiliary results before deriving estimates of Lemmas 4.4 and 4.5, we will state three lemmas whose proofs can be adopted from those in [5], [29] and [8].

Using similar arguments as those in proving [5], Lemma 3.4, we can establish the following lemma.

Lemma 4.1. For any $h \in \mathcal{H}_{p} \cap L_{p^{\prime}}\left(v_{T}\right), p^{\prime} \in(0, p]$, there exists a constant $C_{h, p, p^{\prime}, N}$ such that

$$
C_{h, p, p^{\prime}, N}:=\sup _{g \in S^{N}} \int_{\mathbb{X}_{T}} h^{p^{\prime}}(s, v)(g(s, v)+1) v(d v) d s<\infty .
$$

For any $h \in \mathcal{H}_{2} \cap L_{2}\left(v_{T}\right)$, there exists a constant $C_{h, N}$ such that

$$
C_{h, N}:=\sup _{g \in S^{N}} \int_{\mathbb{X}_{T}} h(s, v)|g(s, v)-1| v(d v) d s<\infty .
$$

Using the argument used for proving [5], Lemmas 3.4 and 3.11, and [29], (3.19), we further get

Lemma 4.2. Let $h: \mathbb{X}_{T} \rightarrow \mathbb{R}$ be a measurable function such that

$$
\int_{\mathbb{X}_{T}}|h(s, v)|^{2} v(d v) d s<\infty
$$

and for all $\delta \in(0, \infty)$

$$
\int_{E} \exp (\delta|h(s, v)|) v(d v) d s<\infty,
$$

for all $E \in \mathcal{B}\left(\mathbb{X}_{T}\right)$ satisfying $\nu_{T}(E)<\infty$.

(a) Fix $N \in \mathbb{N}$, and let $g_{n}, g \in S^{N}$ be such that $g_{n} \rightarrow g$ as $n \rightarrow \infty$. Then

$$
\lim _{n \rightarrow \infty} \int_{\mathbb{X}_{T}} h(s, v)\left(g_{n}(s, v)-1\right) v(d v) d s=\int_{\mathbb{X}_{T}} h(s, v)(g(s, v)-1) v(d v) d s .
$$

(b) Fix $N \in \mathbb{N}$. Given $\varepsilon>0$, there exists a compact set $K_{\varepsilon} \subset \mathbb{X}$, such that

$$
\sup _{g \in S^{N}} \int_{[0, T]} \int_{K_{\varepsilon}^{c}}|h(s, v)||g(s, v)-1| v(d v) d s \leq \varepsilon .
$$

(c) For every $\eta>0$, there exists $\delta>0$, we have such that for any $A \in \mathcal{B}([0, T])$ satisfying $\lambda_{T}(A)<\delta$

$$
\sup _{g \in S^{N}} \int_{A} \int_{\mathbb{X}} h(s, v)|g(s, v)-1| v(d v) d s \leq \eta
$$


Fix $N \in \mathbb{N}$. For any $\varphi_{\varepsilon} \in \tilde{\mathbb{A}}^{N}$, consider the following controlled SPDEs

$$
\begin{aligned}
d \widetilde{X}_{t}^{\varepsilon}= & \mathcal{A}\left(t, \widetilde{X}_{t}^{\varepsilon}\right) d t+\int_{\mathbb{X}} f\left(t, \widetilde{X}_{t}^{\varepsilon}, z\right)\left(\varphi_{\varepsilon}(t, z)-1\right) v(d z) d t \\
& +\varepsilon \int_{\mathbb{X}} f\left(t, \widetilde{X}_{t-}^{\varepsilon}, z\right) \widetilde{N}^{\varepsilon^{-1} \varphi_{\varepsilon}}(d z, d t)
\end{aligned}
$$

with initial condition $\widetilde{X}_{0}^{\varepsilon}=x$.

Recall $\tilde{\mathbb{A}}^{N}$ in Theorem 2.2. Let $\vartheta_{\varepsilon}=\frac{1}{\varphi_{\varepsilon}}$. The following lemma follows from Lemma 2.3 and Section 5.2 in [8]. Recall the notations in Section 2.1, we have the following lemma.

\section{Lemma 4.3.}

$$
\begin{aligned}
\mathcal{E}_{t}^{\varepsilon}\left(\vartheta_{\varepsilon}\right):= & \exp \left\{\int_{(0, t] \times \mathbb{X} \times\left[0, \varepsilon^{-1} \varphi_{\varepsilon}\right]} \log \left(\vartheta_{\varepsilon}(s, x)\right) \bar{N}(d s d x d r)\right. \\
& \left.+\int_{(0, t] \times \mathbb{X} \times\left[0, \varepsilon^{-1} \varphi_{\varepsilon}\right]}\left(-\vartheta_{\varepsilon}(s, x)+1\right) \bar{\nu}_{T}(d s d x d r)\right\} .
\end{aligned}
$$

Consequently,

$$
\mathbb{Q}_{t}^{\varepsilon}(G)=\int_{G} \mathcal{E}_{t}^{\varepsilon}\left(\vartheta_{\varepsilon}\right) d \overline{\mathbb{P}}, \quad \text { for } G \in \mathcal{B}(\overline{\mathbb{M}})
$$

defines a probability measure on $\overline{\mathrm{M}}$.

By the fact that $\varepsilon N^{\varepsilon^{-1}} \varphi_{\varepsilon}$ under $\mathbb{Q}_{T}^{\varepsilon}$ has the same law as that of $\varepsilon N^{\varepsilon^{-1}}$ under $\overline{\mathbb{P}}$. From Theorem 1.1, we see that there exists a unique solution $\widetilde{X}^{\varepsilon}$ to the controlled SPDE (4.4) which satisfies (2) in Theorem 1.1.

By the definition of $\mathcal{G}^{\varepsilon}$, we have

$$
\widetilde{X}^{\varepsilon}=\mathcal{G}^{\varepsilon}\left(\varepsilon N^{\varepsilon^{-1} \varphi_{\varepsilon}}\right)
$$

The following estimates (Lemmas 4.4 and 4.5) will be useful.

Lemma 4.4. For $p=2,2+\beta$ or $\Upsilon$ in (H5), there exists $\varepsilon_{p}, C_{p}>0$ such that

$$
\sup _{\varepsilon \in\left(0, \varepsilon_{p}\right]} \mathbb{E}\left(\sup _{t \in[0, T]}\left\|\tilde{X}_{t}^{\varepsilon}\right\|_{H}^{p}\right)+\mathbb{E}\left(\int_{0}^{T}\left\|\tilde{X}_{t}^{\varepsilon}\right\|_{H}^{p-2}\left\|\tilde{X}_{t}^{\varepsilon}\right\|_{V}^{\alpha} d t\right) \leq C_{p} .
$$

Proof. By Itô's formula, we have

$$
\left\|\tilde{X}_{t}^{\varepsilon}\right\|_{H}^{p}=\|x\|_{H}^{p}+I_{1}(t)+I_{2}(t)+I_{3}(t)+I_{4}(t),
$$


where

$$
\begin{aligned}
I_{1}(t)= & \frac{p}{2} \int_{0}^{t}\left\|\tilde{X}_{s}^{\varepsilon}\right\|_{H}^{p-2}\left(2\left\langle\mathcal{A}\left(s, \tilde{X}_{s}^{\varepsilon}\right), \tilde{X}_{s}^{\varepsilon}\right\rangle_{V^{*}, V}\right) d s \\
I_{2}(t)= & \int_{0}^{t} \int_{\mathbb{X}} p\left\|\tilde{X}_{s-}^{\varepsilon}\right\|_{H}^{p-2}\left\langle\varepsilon f\left(s, \tilde{X}_{s-}^{\varepsilon}, z\right), \tilde{X}_{s-}^{\varepsilon}\right\rangle_{H, H} \tilde{N}^{\varepsilon^{-1} \varphi_{\varepsilon}}(d z, d s), \\
I_{3}(t)= & \int_{0}^{t} \int_{\mathbb{X}}\left[\left\|\tilde{X}_{s-}^{\varepsilon}+\varepsilon f\left(s, \tilde{X}_{s-}^{\varepsilon}, z\right)\right\|_{H}^{p}-\left\|\tilde{X}_{s-}^{\varepsilon}\right\|_{H}^{p}\right. \\
& \left.-p\left\|\tilde{X}_{s-}^{\varepsilon}\right\|_{H}^{p-2}\left\langle\varepsilon f\left(s, \tilde{X}_{s-}^{\varepsilon}, z\right), \tilde{X}_{s-}^{\varepsilon}\right\rangle_{H, H}\right] N^{\varepsilon^{-1} \varphi_{\varepsilon}}(d z, d s),
\end{aligned}
$$

and

$$
I_{4}(t)=p \int_{0}^{t}\left\|\tilde{X}_{s}^{\varepsilon}\right\|_{H}^{p-2}\left\langle\int_{\mathbb{X}} f\left(s, \tilde{X}_{s}^{\varepsilon}, z\right)\left(\varphi_{\varepsilon}(s, z)-1\right), \tilde{X}_{s}^{\varepsilon}\right\rangle_{H, H} v(d z) d s .
$$

Note that by (H3),

$$
\begin{aligned}
I_{1}(t) \leq & \frac{p}{2} \int_{0}^{t}\left\|\tilde{X}_{s}^{\varepsilon}\right\|_{H}^{p-2}\left(F_{S}+F_{S}\left\|\tilde{X}_{S}^{\varepsilon}\right\|_{H}^{2}-\theta\left\|\tilde{X}_{S}^{\varepsilon}\right\|_{V}^{\alpha}\right) d s \\
\leq & -\frac{\theta p}{2} \int_{0}^{t}\left\|\tilde{X}_{s}^{\varepsilon}\right\|_{H}^{p-2}\left\|\tilde{X}_{s}^{\varepsilon}\right\|_{V}^{\alpha} d s \\
& +\frac{p}{2} \int_{0}^{t}\left[\left(\left\|\tilde{X}_{s}^{\varepsilon}\right\|_{H}^{p}+1\right) F_{S}+F_{S}\left\|\tilde{X}_{s}^{\varepsilon}\right\|_{H}^{p}\right] d s \\
\leq & -\frac{\theta p}{2} \int_{0}^{t}\left\|\tilde{X}_{s}^{\varepsilon}\right\|_{H}^{p-2}\left\|\tilde{X}_{s}^{\varepsilon}\right\|_{V}^{\alpha} d s \\
& +\frac{p}{2} \int_{0}^{t} F_{S} d s+\int_{0}^{t} p F_{S}\left\|\tilde{X}_{s}^{\varepsilon}\right\|_{H}^{p} d s
\end{aligned}
$$

and by (H5),

$$
\begin{aligned}
I_{4}(t) \leq & p \int_{0}^{t}\left\|\tilde{X}_{s}^{\varepsilon}\right\|_{H}^{p-1} \int_{\mathbb{X}}\left\|f\left(s, \tilde{X}_{s}^{\varepsilon}, z\right)\right\|_{H}\left|\left(\varphi_{\varepsilon}(s, z)-1\right)\right| v(d z) d s \\
\leq & p \int_{0}^{t}\left\|\tilde{X}_{s}^{\varepsilon}\right\|_{H}^{p-1}\left(1+\left\|\tilde{X}_{s}^{\varepsilon}\right\|_{H}\right) \int_{\mathbb{X}} L_{f}(s, z)\left|\left(\varphi_{\varepsilon}(s, z)-1\right)\right| v(d z) d s \\
\leq & p \int_{0}^{t} \int_{\mathbb{X}} L_{f}(s, z)\left|\left(\varphi_{\varepsilon}(s, z)-1\right)\right| v(d z) d s \\
& +2 p \int_{0}^{t}\left\|\tilde{X}_{S}^{\varepsilon}\right\|_{H}^{p} \int_{\mathbb{X}} L_{f}(s, z)\left|\left(\varphi_{\varepsilon}(s, z)-1\right)\right| v(d z) d s .
\end{aligned}
$$


By Gronwall's inequality, combining (4.6) (4.7), (4.8) and Lemma 4.1,

$$
\begin{aligned}
\left\|\tilde{X}_{t}^{\varepsilon}\right\|_{H}^{p}+\frac{\theta p}{2} \int_{0}^{t}\left\|\tilde{X}_{s}^{\varepsilon}\right\|_{H}^{p-2}\left\|\tilde{X}_{s}^{\varepsilon}\right\|_{V}^{\alpha} d s \\
\leq \\
\quad \exp \left(p \int_{0}^{T} F_{s} d s+2 p C_{L_{f}, N}\right) \\
\quad \times\left(\|x\|_{H}^{p}+\frac{p}{2} \int_{0}^{T} F_{s} d s+\sup _{s \in[0, t]}\left|I_{2}(s)\right|+p C_{L_{f}, N}\right. \\
\quad+\int_{0}^{t} \int_{\mathbb{X}} c_{p}\left(\left\|\tilde{X}_{s-}^{\varepsilon}\right\|_{H}^{p-2}\left\|\varepsilon f\left(s, \tilde{X}_{s-}^{\varepsilon}, z\right)\right\|_{H}^{2}\right. \\
\left.\left.\quad+\left\|\varepsilon f\left(s, \tilde{X}_{s-}^{\varepsilon}, z\right)\right\|_{H}^{p}\right) N^{\varepsilon^{-1} \varphi_{\varepsilon}}(d z, d s)\right)
\end{aligned}
$$

we have used (4.9) in [4] to $I_{3}$, that is,

$$
\left|\|x+h\|_{H}^{p}-\|x\|_{H}^{p}-p\|x\|_{H}^{p-2}\langle x, h\rangle_{H, H}\right| \leq c_{p}\left(\|x\|_{H}^{p-2}\|h\|_{H}^{2}+\|h\|_{H}^{p}\right), \quad \forall x, h \in H .
$$

By Lemma 4.1, we have

$$
\begin{aligned}
& \mathbb{E}\left(\sup _{s \in[0, T]}\left|I_{2}(s)\right|\right) \\
& \leq \mathbb{E}\left(\int _ { 0 } ^ { T } \int _ { \mathbb { X } } \varepsilon ^ { 2 } p ^ { 2 } \| \widetilde { X } ^ { \varepsilon } ( s - ) \| _ { H } ^ { 2 p - 4 } \left\langlef\left(s, \widetilde{X}_{s-}^{\varepsilon}, z\right),\right.\right. \\
& \left.\left.\widetilde{X}^{\varepsilon}(s-)\right|_{H, H} ^{2} N^{\varepsilon^{-1}} \varphi_{\varepsilon}(d z, d s)\right)^{1 / 2} \\
& \leq \mathbb{E}\left(\int_{0}^{T} \int_{\mathbb{X}} \varepsilon^{2} p^{2}\left\|\tilde{X}^{\varepsilon}(s-)\right\|_{H}^{2 p-2} L_{f}^{2}(s, z)\left(\left\|\widetilde{X}_{s-}^{\varepsilon}\right\|_{H}+1\right)^{2} N^{\varepsilon^{-1} \varphi_{\varepsilon}}(d z, d s)\right)^{1 / 2} \\
& \leq \mathbb{E}\left(\sup _{s \in[0, T]}\left\|\tilde{X}_{s}^{\varepsilon}\right\|_{H}^{p}\right. \\
& \left.\cdot \varepsilon^{2} p^{2} \int_{0}^{T} \int_{\mathbb{X}}\left\|\widetilde{X}_{s-}^{\varepsilon}\right\|_{H}^{p-2} L_{f}^{2}(s, z)\left(\left\|\widetilde{X}_{s-}^{\varepsilon}\right\|_{H}+1\right)^{2} N^{\varepsilon^{-1} \varphi_{\varepsilon}}(d z, d s)\right)^{1 / 2} \\
& \leq \frac{1}{4} \mathbb{E}\left(\sup _{s \in[0, T]}\left\|\tilde{X}_{S}^{\varepsilon}\right\|_{H}^{p}\right) \\
& +16 \varepsilon p^{2} \mathbb{E}\left[\left(\sup _{s \in[0, T]}\left\|\tilde{X}_{s}^{\varepsilon}\right\|_{H}^{p}+1\right) \int_{0}^{T} \int_{\mathbb{X}} L_{f}^{2}(s, z) \varphi_{\varepsilon}(s, z) v(d z) d s\right] \\
& \leq\left(\frac{1}{4}+16 \varepsilon p^{2} C_{L_{f}, 2,2, N}\right) \mathbb{E}\left(\sup _{s \in[0, T]}\left\|\tilde{X}_{s}^{\varepsilon}\right\|_{H}^{p}\right)+16 \varepsilon p^{2} C_{L_{f}, 2,2, N} .
\end{aligned}
$$


On the other hand, by Lemma 4.1 again, we have

$$
\begin{aligned}
& \mathbb{E}\left(\int_{0}^{T} \int_{\mathbb{X}} c_{p}\left\|\tilde{X}_{s}^{\varepsilon}\right\|_{H}^{p-2}\left\|\varepsilon f\left(s, \tilde{X}_{s}^{\varepsilon}, z\right)\right\|_{H}^{2} N^{\varepsilon^{-1} \varphi_{\varepsilon}}(d z, d s)\right) \\
& \quad \leq \varepsilon c_{p} \mathbb{E}\left(\int_{0}^{T} \int_{\mathbb{X}}\left\|\tilde{X}_{s}^{\varepsilon}\right\|_{H}^{p-2} L_{f}^{2}(s, z)\left(\left\|\tilde{X}_{s}^{\varepsilon}\right\|_{H}+1\right)^{2} \varphi_{\varepsilon}(s, z) v(d z) d s\right) \\
& \quad \leq \varepsilon c_{p} \mathbb{E}\left[\left(\sup _{s \in[0, T]}\left\|\tilde{X}_{s}^{\varepsilon}\right\|_{H}^{p}+1\right) \int_{0}^{T} \int_{\mathbb{X}} L_{f}^{2}(s, z) \varphi_{\varepsilon}(s, z) v(d z) d s\right] \\
& \quad \leq \varepsilon c_{p} C_{L_{f}, p, p, N} \mathbb{E}\left(\sup _{s \in[0, T]}\left\|\tilde{X}_{s}^{\varepsilon}\right\|_{H}^{p}\right)+\varepsilon c_{p} C_{L_{f}, 2,2, N},
\end{aligned}
$$

and

$$
\begin{aligned}
& \mathbb{E}\left(\int_{0}^{T} \int_{\mathbb{X}} c_{p}\left\|\varepsilon f\left(s, \tilde{X}_{s}^{\varepsilon}, z\right)\right\|_{H}^{p} N^{\varepsilon^{-1} \varphi_{\varepsilon}}(d z, d s)\right) \\
& \quad=\varepsilon^{p-1} c_{p} \mathbb{E}\left(\int_{0}^{T} \int_{\mathbb{X}}\left\|f\left(s, \tilde{X}_{s}^{\varepsilon}, z\right)\right\|_{H}^{p} \varphi_{\varepsilon}(s, z) v(d z) d s\right) \\
& \quad \leq \varepsilon^{p-1} c_{p} \mathbb{E}\left[\left(\sup _{s \in[0, T]}\left\|\tilde{X}_{s}^{\varepsilon}\right\|_{H}^{p}+1\right) \int_{0}^{T} \int_{\mathbb{X}} L_{f}^{p}(s, z) \varphi_{\varepsilon}(s, z) v(d z) d s\right] \\
& \quad \leq \varepsilon^{p-1} c_{p} C_{L_{f}, p, p, N} \mathbb{E}\left(\sup _{s \in[0, T]}\left\|\tilde{X}_{s}^{\varepsilon}\right\|_{H}^{p}\right)+\varepsilon^{p-1} c_{p} C_{L_{f}, p, p, N} .
\end{aligned}
$$

Combining (4.9)-(4.12), we obtain that there exists $\varepsilon_{p}>0$ such that

$$
\begin{aligned}
& \sup _{\varepsilon \in\left(0, \varepsilon_{p}\right]}\left[\mathbb{E}\left(\sup _{s \in[0, T]}\left\|\tilde{X}_{s}^{\varepsilon}\right\|_{H}^{p}\right)+\frac{\theta p}{2} \mathbb{E}\left(\int_{0}^{T}\left\|\tilde{X}_{s}^{\varepsilon}\right\|_{H}^{p-2}\left\|\tilde{X}_{s}^{\varepsilon}\right\|_{V}^{\alpha} d s\right)\right] \\
& \quad \leq C_{N, p, T,\|x\|_{H}, \int_{0}^{T} F_{s} d s, L_{f}} .
\end{aligned}
$$

The proof is complete.

Lemma 4.5. For $p=\frac{\Upsilon}{2}$, there exist $C_{p}$ such that

$$
\sup _{\varepsilon \in\left(0, \varepsilon_{2 p}\right]} \mathbb{E}\left(\int_{0}^{T}\left\|\tilde{X}_{s}^{\varepsilon}\right\|_{V}^{\alpha} d s\right)^{p} \leq C_{p} .
$$

Here $\varepsilon_{2 p}$ comes from Lemma 4.4 . 
Proof. Consider $p=2$ in (4.9), we have

$$
\begin{aligned}
& \theta \int_{0}^{t}\left\|\tilde{X}_{s}^{\varepsilon}\right\|_{V}^{\alpha} d s \\
& \quad \leq C_{N, T, \int_{0}^{T} F_{s} d s, L_{f}}\left(\|x\|_{H}^{2}+\int_{0}^{T} F_{s} d s+\sup _{s \in[0, t]}\left|I_{2}(s)\right|+2 C_{L_{f}, N}+J(t)\right),
\end{aligned}
$$

where

$$
J(t)=\int_{0}^{t} \int_{\mathbb{X}} c_{2}\left(\left\|\varepsilon f\left(s, \widetilde{X}_{s-}^{\varepsilon}, z\right)\right\|_{H}^{2}\right) N^{\varepsilon^{-1} \varphi_{\varepsilon}}(d z, d s) .
$$

In the following calculations, we take $p=\frac{\Upsilon}{2}$. Note that

$$
\begin{aligned}
\mathbb{E}\left(|J(t)|^{p}\right) \leq & c_{p} \mathbb{E}\left(\left|\int_{0}^{T} \int_{\mathbb{X}}\left(\left\|\varepsilon f\left(s, \widetilde{X}_{s-}^{\varepsilon}, z\right)\right\|_{H}^{2}\right) \widetilde{N}^{\varepsilon^{-1} \varphi_{\varepsilon}}(d z, d s)\right|^{p}\right) \\
& +c_{p} \mathbb{E}\left(\left|\int_{0}^{T} \int_{\mathbb{X}}\left(\varepsilon\left\|f\left(s, \widetilde{X}_{s}^{\varepsilon}, z\right)\right\|_{H}^{2}\right) \varphi_{\varepsilon}(s, z) v(d z) d s\right|^{p}\right) .
\end{aligned}
$$

By Kunita's first inequality (refer to Theorem 4.4.23 in [1]), we can continue with

$$
\begin{aligned}
\mathbb{E}\left(|J(t)|^{p}\right) \leq & c_{p} \varepsilon^{2 p-1} \mathbb{E}\left(\int_{0}^{T} \int_{\mathbb{X}}\left\|f\left(s, \widetilde{X}_{s}^{\varepsilon}, z\right)\right\|_{H}^{2 p} \varphi_{\varepsilon}(s, z) v(d z) d s\right) \\
& +c_{p} \varepsilon^{3 p / 2} \mathbb{E}\left(\int_{0}^{T} \int_{\mathbb{X}}\left\|f\left(s, \tilde{X}_{s}^{\varepsilon}, z\right)\right\|_{H}^{4} \varphi_{\varepsilon}(s, z) v(d z) d s\right)^{p / 2} \\
& +c_{p} \varepsilon^{p} \mathbb{E}\left(\int_{0}^{T} \int_{\mathbb{X}}\left\|f\left(s, \widetilde{X}_{s}^{\varepsilon}, z\right)\right\|_{H}^{2} \varphi_{\varepsilon}(s, z) v(d z) d s\right)^{p}
\end{aligned}
$$

Thus, by Lemma 4.1, we have

$$
\begin{aligned}
& \mathbb{E}\left(|J(t)|^{p}\right) \\
& \leq c_{p} \mathbb{E}\left(1+\sup _{s \in[0, T]}\left\|\tilde{X}_{s}^{\varepsilon}\right\|_{H}\right)^{2 p}\left(\varepsilon^{2 p-1} \sup _{\varphi \in S^{N}} \int_{0}^{T} \int_{\mathbb{X}} L_{f}^{2 p}(s, z) \varphi(s, z) v(d z) d s\right. \\
& \quad+\varepsilon^{3 p / 2}\left(\sup _{\varphi \in S^{N}} \int_{0}^{T} \int_{\mathbb{X}} L_{f}^{4}(s, z) \varphi(s, z) v(d z) d s\right)^{p / 2} \\
& \left.\quad+\varepsilon^{p}\left(\sup _{\varphi \in S^{N}} \int_{0}^{T} \int_{\mathbb{X}} L_{f}^{2}(s, z) \varphi(s, z) v(d z) d s\right)^{p}\right) \\
& \leq c_{p} \mathbb{E}\left(1+\sup _{s \in[0, T]}\left\|\widetilde{X}_{s}^{\varepsilon}\right\|\right)^{2 p}\left(\varepsilon^{2 p-1} C_{L_{f}, 2 p, 2 p, N}\right. \\
& \left.\quad+\varepsilon^{3 p / 2}\left(C_{L_{f}, 4,4, N}\right)^{p / 2}+\varepsilon^{p}\left(C_{L_{f}, 2,2, N}\right)^{p}\right) .
\end{aligned}
$$


By Kunita's first inequality again,

$$
\begin{aligned}
& \mathbb{E}\left(\sup _{s \in[0, T]}\left|I_{2}(s)\right|^{p}\right) \\
& \leq c_{p} \varepsilon^{p-1} \mathbb{E}\left(\int_{0}^{T} \int_{\mathbb{X}}\left|\left\langle f\left(s, \tilde{X}_{s}^{\varepsilon}, z\right), \tilde{X}_{s}^{\varepsilon}\right\rangle_{H, H}\right|^{p} \varphi_{\varepsilon}(s, z) v(d z) d s\right) \\
& \quad+c_{p} \varepsilon^{p / 2} \mathbb{E}\left(\int_{0}^{T} \int_{\mathbb{X}}\left|\left\langle f\left(s, \tilde{X}_{s}^{\varepsilon}, z\right), \tilde{X}_{s}^{\varepsilon}\right\rangle_{H, H}\right|^{2} \varphi_{\varepsilon}(s, z) v(d z) d s\right)^{p / 2} \\
& \leq c_{p} \varepsilon^{p-1} \mathbb{E}\left(\int_{0}^{T} \int_{\mathbb{X}}\left\|\tilde{X}_{s}^{\varepsilon}\right\|_{H}^{p} L_{f}^{p}(s, z)\left(1+\left\|\tilde{X}_{s}^{\varepsilon}\right\|_{H}\right)^{p} \varphi_{\varepsilon}(s, z) v(d z) d s\right) \\
& \quad+c_{p} \varepsilon^{p / 2} \mathbb{E}\left(\int_{0}^{T} \int_{\mathbb{X}}\left\|\tilde{X}_{s}^{\varepsilon}\right\|_{H}^{2} L_{f}^{2}(s, z)\left(1+\left\|\tilde{X}_{s}^{\varepsilon}\right\|_{H}\right)^{2} \varphi_{\varepsilon}(s, z) v(d z) d s\right)^{p / 2} \\
& \leq c_{p} \varepsilon^{p-1} \mathbb{E}\left(1+\sup _{s \in[0, T]}\left\|\tilde{X}_{s}^{\varepsilon}\right\|_{H}\right)^{2 p} \sup _{\varphi \in S^{N}} \int_{0}^{T} \int_{\mathbb{X}} L_{f}^{p}(s, z) \varphi(s, z) v(d z) d s \\
&+c_{p} \varepsilon^{p / 2} \mathbb{E}\left(1+\sup _{s \in[0, T]}\left\|\tilde{X}_{s}^{\varepsilon}\right\|_{H}\right)^{2 p}\left(\sup _{\varphi \in S^{N}} \int_{0}^{T} \int_{\mathbb{X}} L_{f}^{2}(s, z) \varphi(s, z) v(d z) d s\right)^{p / 2} \\
& \leq c_{p} \mathbb{E}\left(1+\sup _{s \in[0, T]}\left\|\tilde{X}_{s}^{\varepsilon}\right\|\right)^{2 p}\left(\varepsilon^{p-1} C_{L_{f}, p, p, N}+\varepsilon^{p / 2}\left(C_{L_{f}, 2,2, N}\right)^{p / 2}\right) .
\end{aligned}
$$

Lemma 4.4 and (4.13)-(4.15) imply this lemma.

Finally, we prove the tightness of $\left\{\tilde{X}^{\varepsilon}\right\}$.

Proposition 4.1. For some $\varepsilon_{0}>0,\left\{\tilde{X}^{\varepsilon}\right\}_{\varepsilon \in\left(0, \varepsilon_{0}\right]}$ is tight in $D\left([0, T], V^{*}\right)$ with the Skorohod topology. Moreover, set

$$
\begin{aligned}
M_{t}^{\varepsilon} & =\int_{0}^{t} \int_{\mathbb{X}} \varepsilon f\left(s, \widetilde{X}_{s-}^{\varepsilon}, z\right) \widetilde{N}^{\varepsilon^{-1} \varphi_{\varepsilon}}(d z, d s), \\
Z_{t}^{\varepsilon} & =\int_{0}^{t} \int_{\mathbb{X}} f\left(s, \widetilde{X}_{s}^{\varepsilon}, z\right)\left(\varphi_{\varepsilon}(s, z)-1\right) v(d z) d s, \\
Y_{t}^{\varepsilon} & =\int_{0}^{t} \mathcal{A}\left(s, \widetilde{X}_{s}^{\varepsilon}\right) d s,
\end{aligned}
$$

then

(a) $\lim _{\varepsilon \rightarrow 0} \mathbb{E}\left(\sup _{t \in[0, T]}\left\|M_{t}^{\varepsilon}\right\|_{H}^{2}\right)=0$,

(b) $\left(Z_{t}^{\varepsilon}\right)_{0 \leq t \leq T}$ is tight in $C\left([0, T], V^{*}\right)$,

(c) $\left(Y_{t}^{\varepsilon}\right)_{0 \leq t \leq T}$ is tight in $C\left([0, T], V^{*}\right)$. 
Proof. (a) By Lemma 4.1, we have

$$
\begin{aligned}
& \mathbb{E}\left(\sup _{t \in[0, T]}\left\|M_{t}^{\varepsilon}\right\|_{H}^{2}\right) \\
& \quad \leq C \varepsilon \mathbb{E}\left(\int_{0}^{T} \int_{\mathbb{X}}\left\|f\left(s, \tilde{X}_{s}^{\varepsilon}, z\right)\right\|_{H}^{2} \varphi_{\varepsilon}(s, z) v(d z) d s\right) \\
& \quad \leq C \varepsilon \mathbb{E}\left(\int_{0}^{T} \int_{\mathbb{X}} L_{f}^{2}(s, z)\left(1+\left\|\tilde{X}_{s}^{\varepsilon}\right\|_{H}\right)^{2} \varphi_{\varepsilon}(s, z) v(d z) d s\right) \\
& \quad \leq C \varepsilon \mathbb{E}\left(1+\sup _{s \in[0, T]}\left\|\tilde{X}_{s}^{\varepsilon}\right\|_{H}\right)^{2}\left(\sup _{\varphi \in S^{N}} \int_{0}^{T} \int_{\mathbb{X}} L_{f}^{2}(s, z) \varphi(s, z) v(d z) d s\right) \\
& \quad \leq C \varepsilon \mathbb{E}\left(1+\sup _{s \in[0, T]}\left\|\tilde{X}_{s}^{\varepsilon}\right\|_{H}\right)^{2} C_{L_{f}, 2,2, N} \\
& \rightarrow 0, \quad \text { as } \varepsilon \rightarrow 0 .
\end{aligned}
$$

(b) It is sufficient to prove that for any $\delta>0$, there exists a compact subset $K_{\delta} \subset C\left([0, T], V^{*}\right)$ such that

$$
\overline{\mathbb{P}}\left(Z^{\varepsilon} \in K_{\delta}\right)>1-\delta
$$

Denote

$$
\begin{aligned}
\mathcal{D}_{M, N} & =\left\{\left(r_{t}, g_{t}\right): r . \in D([0, T], H) \cap L^{\alpha}([0, T], V), \sup _{t \in[0, T]}\left\|r_{t}\right\|_{H} \leq M ; g \in S^{N}\right\}, \\
\mathcal{R}\left(\mathcal{D}_{M, N}\right) & =\left\{y .=\int_{0} \int_{\mathbb{X}} f\left(s, r_{s}, z\right)(g(s, z)-1) v(d z) d s,(r, g) \in \mathcal{D}_{M, N}\right\} .
\end{aligned}
$$

For any $y \in \mathcal{R}\left(\mathcal{D}_{M, N}\right)$, we have

$$
\begin{aligned}
\left\|y_{t}-y_{s}\right\|_{H} & \leq \int_{s}^{t} \int_{\mathbb{X}}\|f(l, r(l), z)\|_{H}|g(l, z)-1| v(d z) d l \\
& \leq \sup _{l \in[s, t]}\left(1+\|r(l)\|_{H}\right) \int_{s}^{t} \int_{\mathbb{X}} L_{f}(l, z)|g(l, z)-1| v(d z) d l \\
& \leq(M+1) \sup _{\varphi \in S^{N}} \int_{s}^{t} \int_{\mathbb{X}} L_{f}(l, z)|\varphi(l, z)-1| v(d z) d l .
\end{aligned}
$$

Applying Lemma 4.1(c) in Lemma 4.2 and (4.17), we obtain the following:

(1) for any $\eta>0$, there exists $\varpi>0$ (independent on $y$ ) such that for any $s, t \in[0, T]$ and $|t-s| \leq \varpi$

$$
\left\|y_{t}-y_{s}\right\|_{H} \leq \eta, \quad \forall y \in \mathcal{R}\left(\mathcal{D}_{M, N}\right)
$$


(2)

$$
\sup _{y \in \mathcal{R}\left(\mathcal{D}_{M, N}\right)} \sup _{t \in[0, T]}\left\|y_{t}\right\|_{H}=\sup _{y \in \mathcal{R}\left(\mathcal{D}_{M, N}\right)} \sup _{t \in[0, T]}\left\|y_{t}-y_{0}\right\|_{H} \leq(M+1) C_{L_{f}, N} .
$$

Since $V \hookrightarrow H$ is compact, we also have $H \hookrightarrow V^{*}$ compactly. By Ascoli-Arzelá's theorem, the complement of $\mathcal{R}\left(\mathcal{D}_{M, N}\right)$ in $C\left([0, T], V^{*}\right)$, denoted by $\overline{\mathcal{R}}\left(\mathcal{D}_{M, N}\right)$, is a compact subset in $C\left([0, T], V^{*}\right)$.

On the other hand,

$$
\begin{aligned}
\overline{\mathbb{P}}\left(Z^{\varepsilon} \in \overline{\mathcal{R}}\left(\mathcal{D}_{M, N}\right)\right) & \geq \overline{\mathbb{P}}\left(\sup _{t \in[0, T]}\left\|\tilde{X}_{t}^{\varepsilon}\right\|_{H} \leq M\right) \\
& =1-\overline{\mathbb{P}}\left(\sup _{t \in[0, T]}\left\|\tilde{X}_{t}^{\varepsilon}\right\|_{H}>M\right) \\
& \geq 1-\mathbb{E}\left(\sup _{t \in[0, T]}\left\|\tilde{X}_{t}^{\varepsilon}\right\|_{H}^{2}\right) / M^{2} \\
& \geq 1-C_{2} / M^{2},
\end{aligned}
$$

we have applied Lemma 4.4 in the last inequality and this establishes that $\left\{Z^{\varepsilon}\right\}$ is tight in $C\left([0, T], V^{*}\right)$.

(c) By Lemmas 4.4 and 4.5, recall $\eta_{0}$ in (H5), let $p=\alpha+\eta_{0}$, we have

$$
\begin{aligned}
\mathbb{E}\left\|Y_{t}^{\varepsilon}-Y_{s}^{\varepsilon}\right\|_{V^{*}}^{p} \leq \mathbb{E}\left|\int_{s}^{t}\left\|\mathcal{A}\left(l, \tilde{X}_{l}^{\varepsilon}\right)\right\|_{V^{*}} d l\right|^{p} \\
\leq|t-s|^{p / \alpha} \mathbb{E}\left(\int_{s}^{t}\left\|\mathcal{A}\left(l, \tilde{X}_{l}^{\varepsilon}\right)\right\|_{V^{*}}^{\frac{\alpha}{\alpha-1}} d l\right)^{\frac{(\alpha-1) p}{\alpha}} \\
\leq|t-s|^{p / \alpha} \mathbb{E}\left(\int_{s}^{t}\left(F_{l}+C\left\|\tilde{X}_{l}^{\varepsilon}\right\|_{V}^{\alpha}\right)\left(1+\left\|\tilde{X}_{l}^{\varepsilon}\right\|_{H}^{\beta}\right) d l\right)^{\frac{(\alpha-1) p}{\alpha}} \\
\leq|t-s|^{p / \alpha}\left[\mathbb{E}\left(\sup _{l \in[0, T]}\left(1+\left\|\tilde{X}_{l}^{\varepsilon}\right\|_{H}^{\beta}\right)^{\frac{2(\alpha-1) p}{\alpha}}\right)\right. \\
\left.\quad+\mathbb{E}\left(\int_{s}^{t} F_{l}+C\left\|\tilde{X}_{l}^{\varepsilon}\right\|_{V}^{\alpha} d l\right)^{\frac{2(\alpha-1) p}{\alpha}}\right] \\
\leq C_{\alpha, p, F|t-s|^{p / \alpha} .}
\end{aligned}
$$

Hence, a direct application of Kolmogorov's criterion, for every $\varpi \in\left(0, \frac{1}{\alpha}-\frac{1}{p}\right)$, there exists constant $C_{\varpi}$ independent on $\varepsilon$ such that

$$
\mathbb{E}\left(\sup _{t \neq s \in[0, T]} \frac{\left\|Y_{t}^{\varepsilon}-Y_{s}^{\varepsilon}\right\|_{V^{*}}^{p}}{|t-s|^{p \varpi}}\right) \leq C_{\varpi} .
$$


On the other hand, by (4.4), we have

$$
\tilde{X}_{t}^{\varepsilon}=x+Y_{t}^{\varepsilon}+Z_{t}^{\varepsilon}+M_{t}^{\varepsilon} .
$$

Then

$$
\begin{aligned}
& \mathbb{E}\left(\sup _{t \in[0, T]}\left\|Y_{t}^{\varepsilon}\right\|_{H}^{2}\right) \\
& \quad \leq C\left[\|x\|_{H}^{2}+\mathbb{E}\left(\sup _{t \in[0, T]}\left\|\tilde{X}_{t}^{\varepsilon}\right\|_{H}^{2}\right)+\mathbb{E}\left(\sup _{t \in[0, T]}\left\|Z_{t}^{\varepsilon}\right\|_{H}^{2}\right)+\mathbb{E}\left(\sup _{t \in[0, T]}\left\|M_{t}^{\varepsilon}\right\|_{H}^{2}\right)\right] .
\end{aligned}
$$

Notice that

$$
\begin{aligned}
& \mathbb{E}\left(\sup _{t \in[0, T]}\left\|Z_{t}^{\varepsilon}\right\|_{H}^{2}\right) \\
& \leq \mathbb{E}\left(\int_{0}^{T} \int_{\mathbb{X}}\left\|f\left(s, \tilde{X}_{s}^{\varepsilon}, z\right)\right\|_{H}\left|\varphi_{\varepsilon}(s, z)-1\right| \nu(d z) d s\right)^{2} \\
& \quad \leq C \mathbb{E}\left(1+\sup _{t \in[0, T]}\left\|\widetilde{X}_{t}^{\varepsilon}\right\|_{H}\right)^{2}\left(\sup _{\varphi \in S^{N}} \int_{0}^{T} \int_{\mathbb{X}} L_{f}(s, z)|\varphi(s, z)-1| v(d z) d s\right)^{2} \\
& \quad \leq C C_{L_{f}, N}^{2} \mathbb{E}\left(1+\sup _{t \in[0, T]}\left\|\tilde{X}_{t}^{\varepsilon}\right\|_{H}\right)^{2} .
\end{aligned}
$$

By Lemma 4.4, (4.19), (4.20) and (4.16), we have

$$
\mathbb{E}\left(\sup _{t \in[0, T]}\left\|Y_{t}^{\varepsilon}\right\|_{H}^{2}\right) \leq C<\infty
$$

where $C$ is independent of $\varepsilon$.

For $\varpi \in(0,1)$ and $R>0$. Set

$$
K_{R, \varpi}:=\left\{j \in C\left([0, T], V^{*}\right): \sup _{t \in[0, T]}\left\|j_{t}\right\|_{H}+\sup _{s \neq t \in[0, T]} \frac{\left\|j_{t}-j_{s}\right\|_{V^{*}}}{|t-s|^{\varpi}} \leq R\right\} .
$$

Since $V \hookrightarrow H$ is compact, we also have $H \hookrightarrow V^{*}$ compactly. By Ascoli-Arzelá's theorem, $K_{R, \varpi}$ is a compact subset of $C\left([0, T], V^{*}\right)$. By (4.18), (4.21) and Chebyshev's inequality, for some $\varpi \in(0,1)$ and any $R>0$, we have

$$
\overline{\mathbb{P}}\left(Y^{\varepsilon} \notin K_{R, \varpi}\right) \geq \frac{C_{T, \varpi}}{R} .
$$

This implies the tightness of $\left\{Y^{\varepsilon}\right\}$ in $C\left([0, T], V^{*}\right)$.

The tightness of $\left\{\widetilde{X}^{\varepsilon}\right\}$ in $D\left([0, T], V^{*}\right)$ then follows from (4.4) and the conclusions proved above. 


\section{Convergence of the processes}

With the tightness result obtained in the last section, the main result of this section is to identify the limit of $\widetilde{X}^{\varepsilon}$ as the solution of the non-random equation (5.27). We use the three parts of $\widetilde{X}^{\varepsilon}$ we utilized in last section. As the limit of $M^{\varepsilon}$ is 0 , we only need to characterize the limits of the other two. Taking $X$ as a limit point of $\widetilde{X}^{\varepsilon}$, we first prove that $Y^{\varepsilon}$ has a limit in Lemma 5.1, and then identify this limit with the desired form in Lemma 5.4 while Lemma 5.3 is a necessary preparation for Lemma 5.4. The limit of $Z^{\varepsilon}$ with the desired form is identified in Lemma 5.2.

Throughout this section, we assume that for almost all $\omega$, as $\varepsilon \rightarrow 0, \varphi_{\varepsilon}(\cdot, \cdot)(\omega)$ converges to $\varphi(\cdot, \cdot)(\omega)$ in $S^{N}$ weakly, and $X^{\varepsilon}(\omega)$ converges to $X(\omega)$ in $D\left([0, T], V^{*}\right)$ strongly with supremum norm.

Set

$$
\mathcal{K}=L^{\alpha}([0, T] \times \Omega \rightarrow V ; d t \times \overline{\mathbb{P}}), \quad \mathcal{K}^{*}=L^{\frac{\alpha}{\alpha-1}}\left([0, T] \times \Omega \rightarrow V^{*} ; d t \times \overline{\mathbb{P}}\right) .
$$

Lemma 5.1. There exists a subsequence $\left(\varepsilon_{k}\right), \bar{X} \in \mathcal{K} \cap L^{\infty}\left([0, T], L^{\beta+2}(\Omega, H)\right)$ and $Y \in \mathcal{K}^{*}$ such that

(i) $X^{\varepsilon_{k}} \rightarrow \bar{X}$ in $\mathcal{K}$ weakly and in $L^{\infty}\left([0, T], L^{\beta+2}(\Omega, H)\right)$ in weak-star topology,

(ii) $\mathcal{A}\left(\cdot, X^{\varepsilon_{k}}\right) \rightarrow Y$ in $\mathcal{K}^{*}$ weakly,

(iii)

$$
\lim _{\varepsilon \rightarrow 0} \mathbb{E}\left(\sup _{t \in[0, T]}\left\|X_{t}^{\varepsilon}-X_{t}\right\|_{V^{*}}\right)=0
$$

and for $m=\frac{\alpha}{\alpha+1}$,

$$
\lim _{\varepsilon \rightarrow 0} \mathbb{E} \int_{0}^{T}\left\|X_{t}^{\varepsilon}-X_{t}\right\|_{H}^{2 m} d t=0 .
$$

Proof. (i) following from Lemma 4.4. For (ii), by Lemma 4.4 again,

$$
\begin{aligned}
\left\|\mathcal{A}\left(\cdot, X^{\varepsilon}(\cdot)\right)\right\|_{\mathcal{K}^{*}}^{\frac{\alpha-1}{\alpha}} & =\mathbb{E}\left(\int_{0}^{T}\left\|\mathcal{A}\left(t, X_{t}^{\varepsilon}\right)\right\|_{V^{*}}^{\frac{\alpha}{\alpha-1}} d t\right) \\
& \leq \mathbb{E}\left(\int_{0}^{T}\left(F_{t}+C\left\|X_{t}^{\varepsilon}\right\|_{V}^{\alpha}\right)\left(1+\left\|X_{t}^{\varepsilon}\right\|_{H}^{\beta}\right) d t\right) \\
& \leq C<\infty .
\end{aligned}
$$

Lemma 4.4 implies

$$
\mathbb{E}\left(\sup _{t \in[0, T]}\left\|X_{t}^{\varepsilon}\right\|_{H}^{2}\right) \leq C_{2, N, x}
$$

and

$$
\mathbb{E}\left(\int_{0}^{T}\left\|X_{t}^{\varepsilon}\right\|_{V}^{\alpha} d t\right) \leq C .
$$


Hence, by the strong convergence of $X^{\varepsilon}(\omega)$ to $X(\omega)$ in $D\left([0, T], V^{*}\right)$ with sup norm, Fatou's lemma, (5.2) and (5.3), we have

$$
\begin{aligned}
& \mathbb{E}\left(\sup _{t \in[0, T]}\left\|X_{t}\right\|_{H}^{2}\right) \leq \liminf _{\varepsilon \rightarrow 0} \mathbb{E}\left(\sup _{t \in[0, T]}\left\|X_{t}^{\varepsilon}\right\|_{H}^{2}\right) \leq C_{2, N, x}, \\
& \mathbb{E}\left(\int_{0}^{T}\left\|X_{t}\right\|_{V}^{\alpha} d t\right) \leq \liminf _{\varepsilon \rightarrow 0} \mathbb{E}\left(\int_{0}^{T}\left\|X_{t}^{\varepsilon}\right\|_{V}^{\alpha} d t\right) \leq C
\end{aligned}
$$

and

$$
\lim _{\varepsilon \rightarrow 0} \mathbb{E}\left(\sup _{t \in[0, T]}\left\|X_{t}^{\varepsilon}-X_{t}\right\|_{V^{*}}\right)=0
$$

Equation (5.6) can be seen as following. Set

$$
\Omega_{\delta}^{\varepsilon}=\left\{\omega: \sup _{t \in[0, T]}\left\|X_{t}^{\varepsilon}-X_{t}\right\|_{V^{*}} \geq \delta\right\}
$$

The strong convergence of $X^{\varepsilon}(\omega)$ to $X(\omega)$ in $D\left([0, T], V^{*}\right)$ with sup norm implies

$$
\lim _{\varepsilon \rightarrow 0} \overline{\mathbb{P}}\left(\Omega_{\delta}^{\varepsilon}\right)=0, \quad \forall \delta>0 .
$$

Applying (5.7), (5.2) and (5.4) to (5.6), we have

$$
\begin{aligned}
& \lim _{\varepsilon \rightarrow 0} \mathbb{E}\left(\sup _{t \in[0, T]}\left\|X_{t}^{\varepsilon}-X_{t}\right\|_{V^{*}}\right) \\
& \quad=\lim _{\varepsilon \rightarrow 0}\left[\mathbb{E}\left(\sup _{t \in[0, T]}\left\|X_{t}^{\varepsilon}-X_{t}\right\|_{V^{*}} \cdot 1_{\Omega_{\delta}^{\varepsilon}}\right)+\mathbb{E}\left(\sup _{t \in[0, T]}\left\|X_{t}^{\varepsilon}-X_{t}\right\|_{V^{*}} \cdot 1_{\left(\Omega_{\delta}^{\varepsilon}\right)^{c}}\right)\right] \\
& \quad \leq \delta+\lim _{\varepsilon \rightarrow 0}\left(\mathbb{E}\left(\sup _{t \in[0, T]}\left\|X_{t}^{\varepsilon}-X_{t}\right\|_{V^{*}}^{2}\right)\right)^{1 / 2} \cdot\left(\overline{\mathbb{P}}\left(\Omega_{\delta}^{\varepsilon}\right)\right)^{1 / 2} \\
& \quad \leq \delta .
\end{aligned}
$$

The arbitrary of $\delta$ implies (5.6).

Taking $m=\frac{\alpha}{\alpha+1}$, we get

$$
\begin{aligned}
\mathbb{E} \int_{0}^{T}\left\|X_{t}^{\varepsilon}-X_{t}\right\|_{H}^{2 m} d t & =\mathbb{E} \int_{0}^{T}\left\langle X_{t}^{\varepsilon}-X_{t}, X_{t}^{\varepsilon}-X_{t}\right\rangle_{V^{*}, V}^{m} d t \\
& \leq \mathbb{E} \int_{0}^{T}\left\|X_{t}^{\varepsilon}-X_{t}\right\|_{V^{*}}^{m}\left\|X_{t}^{\varepsilon}-X_{t}\right\|_{V}^{m} d t \\
& \leq\left(\mathbb{E} \int_{0}^{T}\left\|X_{t}^{\varepsilon}-X_{t}\right\|_{V^{*}} d t\right)^{\frac{\alpha-m}{\alpha}}\left(\mathbb{E} \int_{0}^{T}\left\|X_{t}^{\varepsilon}-X_{t}\right\|_{V}^{\alpha} d t\right)^{\frac{m}{\alpha}}
\end{aligned}
$$


Combining (5.3), (5.5) and (5.6), we have

$$
\lim _{\varepsilon \rightarrow 0} \mathbb{E} \int_{0}^{T}\left\|X_{t}^{\varepsilon}-X_{t}\right\|_{H}^{2 m} d t=0 .
$$

Lemma 5.2. For any $h \in H$, we have

$$
\begin{gathered}
\lim _{\varepsilon_{k} \rightarrow 0}\left\langle\int_{0}^{t} \int_{\mathbb{X}} f\left(s, X_{s}^{\varepsilon_{k}}, z\right)\left(\varphi_{\varepsilon_{k}}(s, z)-1\right) v(d z) d s, h\right\rangle_{H, H} \\
=\left\langle\int_{0}^{t} \int_{\mathbb{X}} f\left(s, X_{s}, z\right)(\varphi(s, z)-1) v(d z) d s, h\right\rangle_{H, H} .
\end{gathered}
$$

Proof. Denote $\zeta(s, z)=\left\langle f\left(s, X_{s}, z\right), h\right\rangle_{H, H}$. Since $\sup _{s \in[0, T]}\left\|X_{s}\right\|_{H}<\infty$, $\overline{\mathbb{P}}$-a.s., and $L_{f} \in$ $\mathcal{H}_{2}$, it follows from Remark 3.1 and Lemma 4.2 that

$$
\begin{gathered}
\lim _{\varepsilon_{k} \rightarrow 0}\left\langle\int_{0}^{t} \int_{\mathbb{X}} f\left(s, X_{s}, z\right)\left(\varphi_{\varepsilon_{k}}(s, z)-1\right) v(d z) d s, h\right\rangle_{H, H} \\
=\left\langle\int_{0}^{t} \int_{\mathbb{X}} f\left(s, X_{s}, z\right)(\varphi(s, z)-1) v(d z) d s, h\right\rangle_{H, H} .
\end{gathered}
$$

For any $\delta>0$, denote $A_{\delta, \varepsilon}(\omega):=\left\{s \in[0, T]:\left\|X_{s}^{\varepsilon}-X_{S}\right\|_{H}>\delta\right\}$. By (5.8)

$$
\lim _{\varepsilon \rightarrow 0} \mathbb{E}\left(\lambda_{T}\left(A_{\delta, \varepsilon}\right)\right) \leq \frac{1}{\delta^{2 m}} \lim _{\varepsilon \rightarrow 0} \mathbb{E} \int_{0}^{T}\left\|X_{t}^{\varepsilon}-X_{t}\right\|_{H}^{2 m} d t=0 .
$$

Therefore, there exists a subsequence $\varepsilon_{k}$ (for simplicity, we still denote it by the same notation $\varepsilon_{k}$ ) such that

$$
\lim _{\varepsilon_{k} \rightarrow 0} \lambda_{T}\left(A_{\delta, \varepsilon_{k}}\right)=0, \quad \overline{\mathbb{P}} \text {-a.s. }
$$

Applying Lemma 4.1, we have

$$
\begin{aligned}
& \int_{0}^{T} \int_{\mathbb{X}}\left\|f\left(s, X_{s}^{\varepsilon_{k}}, z\right)-f\left(s, X_{s}, z\right)\right\|_{H}\left|\varphi_{\varepsilon_{k}}(s, z)-1\right| v(d z) d s \\
& \quad \leq \int_{0}^{T} \int_{\mathbb{X}} G_{f}(s, z)\left\|X_{s}^{\varepsilon_{k}}-X_{s}\right\|_{H}\left|\varphi_{\varepsilon_{k}}(s, z)-1\right| v(d z) d s \\
& \quad \leq \delta \int_{A_{\delta, \varepsilon_{k}}^{c}} \int_{\mathbb{X}} G_{f}(s, z)\left|\varphi_{\varepsilon_{k}}(s, z)-1\right| v(d z) d s \\
& \quad+\sup _{s \in[0, T]}\left\|X_{s}^{\varepsilon_{k}}-X_{s}\right\|_{H} \int_{A_{\delta, \varepsilon_{k}}} \int_{\mathbb{X}} G_{f}(s, z)\left|\varphi_{\varepsilon_{k}}(s, z)-1\right| v(d z) d s
\end{aligned}
$$




$$
\begin{aligned}
\leq & \delta \sup _{\varphi \in S^{N}} \int_{0}^{T} \int_{\mathbb{X}} G_{f}(s, z)|\varphi(s, z)-1| v(d z) d s \\
& +\sup _{s \in[0, T]}\left\|X_{s}^{\varepsilon_{k}}-X_{s}\right\|_{H} \sup _{\varphi \in S^{N}} \int_{A_{\delta, \varepsilon_{k}}} \int_{\mathbb{X}} G_{f}(s, z)|\varphi(s, z)-1| v(d z) d s \\
\leq & \delta C_{G_{f}, N}+\sup _{s \in[0, T]}\left\|X_{s}^{\varepsilon_{k}}-X_{S}\right\|_{H} \sup _{\varphi \in S^{N}} \int_{A_{\delta, \varepsilon_{k}}} \int_{\mathbb{X}} G_{f}(s, z)|\varphi(s, z)-1| v(d z) d s .
\end{aligned}
$$

Notice that

$$
\begin{aligned}
\mathbb{E}\left(\sup _{s \in[0, T]}\left\|X_{s}^{\varepsilon_{k}}-X_{s}\right\|_{H} \sup _{\varphi \in S^{N}} \int_{A_{\delta, \varepsilon_{k}}} \int_{\mathbb{X}} G_{f}(s, z)|\varphi(s, z)-1| v(d z) d s\right) \\
\leq\left(\mathbb{E}\left(\sup _{s \in[0, T]}\left\|X_{s}^{\varepsilon_{k}}-X_{s}\right\|_{H}^{2}\right)\right)^{\frac{1}{2}} \\
\quad \times\left(\mathbb{E}\left(\sup _{\varphi \in S^{N}} \int_{A_{\delta, \varepsilon_{k}}} \int_{\mathbb{X}} G_{f}(s, z)|\varphi(s, z)-1| v(d z) d s\right)^{2}\right)^{\frac{1}{2}} .
\end{aligned}
$$

By the dominated convergence theorem, Lemma 4.2(c) and Lemma 4.1, we have

$$
\lim _{\varepsilon_{k} \rightarrow 0} \mathbb{E}\left(\sup _{\varphi \in S^{N}} \int_{A_{\delta, \varepsilon_{k}}} \int_{\mathbb{X}} G_{f}(s, z)|\varphi(s, z)-1| v(d z) d s\right)^{2}=0 .
$$

Hence, (5.2), (5.4), (5.12)-(5.14) imply

$$
\lim _{\varepsilon_{k} \rightarrow 0} \mathbb{E}\left(\int_{0}^{T} \int_{\mathbb{X}}\left\|f\left(s, X_{s}^{\varepsilon_{k}}, z\right)-f\left(s, X_{s}, z\right)\right\|_{H}\left|\varphi_{\varepsilon_{k}}(s, z)-1\right| v(d z) d s\right)=0 .
$$

So, there exists a subsequence $\varepsilon_{k}$ (for simplicity, we still denote it by the same notation $\varepsilon_{k}$ ) such that

$$
\lim _{\varepsilon_{k} \rightarrow 0} \int_{0}^{T} \int_{\mathbb{X}}\left\|f\left(s, X_{s}^{\varepsilon_{k}}, z\right)-f\left(s, X_{s}, z\right)\right\|_{H}\left|\varphi_{\varepsilon_{k}}(s, z)-1\right| v(d z) d s=0, \quad \overline{\mathbb{P}} \text {-a.s. }
$$

Combining this with (5.10), we arrive at (5.9).

Define

$$
\widetilde{X}_{t}:=x+\int_{0}^{t} Y_{s} d s+\int_{0}^{t} \int_{\mathbb{X}} f\left(s, X_{s}, z\right)(\varphi(s, z)-1) v(d z) d s .
$$

By taking weak limit of (4.4), it is not difficulty to see that

$$
\widetilde{X}_{t}(\omega)=\bar{X}_{t}(\omega)=X_{t}(\omega), \quad \text { for } d t \times \overline{\mathbb{P}} \text {-almost all }(t, \omega)
$$


Set

$$
\mathcal{N}:=\left\{\phi: \phi \text { is a } V \text {-valued } \overline{\mathcal{F}}_{t} \text {-adapted process such that } \mathbb{E}\left(\int_{0}^{T} \rho\left(\phi_{s}\right) d s\right)<\infty\right\} .
$$

Fix $\phi \in \mathcal{K} \cap \mathcal{N} \cap L^{\infty}\left([0, T], L^{\beta+2}(\Omega, H)\right)$ and $\psi \in L^{\infty}([0, T], \mathbb{R})$. Denote

$$
\begin{aligned}
G(X, \varphi, Y):= & \mathbb{E}\left[\int_{0}^{T} \psi_{t} \int_{0}^{t} e^{-\int_{0}^{s}\left(K_{l}+\rho\left(\phi_{l}\right)\right) d l}\right. \\
& \left.\times 2\left\langle\int_{\mathbb{X}} f\left(s, X_{s}, z\right)(\varphi(s, z)-1) v(d z), Y_{s}\right\rangle_{H, H} d s d t\right] .
\end{aligned}
$$

The following limiting result will be needed later.

\section{Lemma 5.3.}

$$
\lim _{\varepsilon_{k} \rightarrow 0} G\left(X^{\varepsilon_{k}}, \varphi_{\varepsilon_{k}}, X^{\varepsilon_{k}}\right)=G(X, \varphi, X)
$$

Proof. For any fixed $(t, \omega) \in[0, T] \times \Omega$. Set

$$
\zeta(s, z)=\psi_{t} e^{-\int_{0}^{s}\left(K_{l}+\rho\left(\phi_{l}\right)\right) d l}\left\langle f\left(s, X_{s}, z\right), X_{s}\right\rangle_{H, H} .
$$

By Lemma 4.2 and $\sup _{s \in[0, T]}\left\|X_{S}\right\|_{H}<\infty \overline{\mathbb{P}}$-a.s., we have $\forall(t, \omega) \in[0, T] \times \Omega$,

$$
\begin{gathered}
\lim _{\varepsilon_{k} \rightarrow 0} \psi_{t} \int_{0}^{t} e^{-\int_{0}^{s}\left(K_{l}+\rho\left(\phi_{l}\right)\right) d l} 2\left\langle\int_{\mathbb{X}} f\left(s, X_{s}, z\right)\left(\varphi_{\varepsilon_{k}}(s, z)-1\right) v(d z), X_{s}\right\rangle_{H, H} d s \\
\quad=\psi_{t} \int_{0}^{t} e^{-\int_{0}^{s}\left(K_{l}+\rho\left(\phi_{l}\right)\right) d l} 2\left\langle\int_{\mathbb{X}} f\left(s, X_{s}, z\right)(\varphi(s, z)-1) v(d z), X_{s}\right\rangle_{H, H} d s .
\end{gathered}
$$

On the other hand, by Lemma 4.1

$$
\begin{aligned}
& \sup _{\varphi \in S^{N}}\left|\psi_{t} \int_{0}^{t} e^{-\int_{0}^{s}\left(K_{l}+\rho\left(\phi_{l}\right)\right) d l}\left(2\left\langle\int_{\mathbb{X}} f\left(s, X_{s}, z\right)(\varphi(s, z)-1) v(d z), X_{S}\right\rangle_{H, H}\right) d s\right| \\
& \leq C_{\psi} \sup _{\varphi \in S^{N}} \int_{0}^{T} \int_{\mathbb{X}}\left\|f\left(s, X_{s}, z\right)\right\|_{H}\left\|X_{s}\right\|_{H}|\varphi(s, z)-1| v(d z) d s \\
& \leq C_{\psi}\left(1+\sup _{s \in[0, T]}\left\|X_{S}\right\|_{H}\right)^{2} \sup _{\varphi \in S^{N}} \int_{0}^{T} \int_{\mathbb{X}} L_{f}(s, z)|\varphi(s, z)-1| v(d z) d s \\
& \quad \leq C_{\psi, L_{f}, N}\left(1+\sup _{s \in[0, T]}\left\|X_{S}\right\|_{H}\right)^{2} .
\end{aligned}
$$


By the dominated convergence theorem, we have

$$
\lim _{\varepsilon_{k} \rightarrow 0} G\left(X, \varphi_{\varepsilon_{k}}, X\right)=G(X, \varphi, X) .
$$

Let $\delta>0$. Recall

$$
A_{\delta, \varepsilon_{k}}:=\left\{s \in[0, T]:\left\|X_{s}^{\varepsilon_{k}}-X_{s}\right\|_{H}>\delta\right\},
$$

and (5.11) that is there exists a subsequence $\varepsilon_{k}$ such that

$$
\lim _{\varepsilon_{k} \rightarrow 0} \lambda_{T}\left(A_{\delta, \varepsilon_{k}}\right)=0, \quad \overline{\mathbb{P}} \text {-a.s. }
$$

Then we have

$$
\begin{aligned}
& \left|G\left(X^{\varepsilon_{k}}, \varphi_{\varepsilon_{k}}, X^{\varepsilon_{k}}\right)-G\left(X^{\varepsilon_{k}}, \varphi_{\varepsilon_{k}}, X\right)\right| \\
& \leq C \mathbb{E}\left(\int_{0}^{T} \int_{\mathbb{X}}\left\|f\left(s, X_{s}^{\varepsilon_{k}}, z\right)\right\|_{H}\left|\varphi_{\varepsilon_{k}}(s, z)-1\right|\left\|X_{s}^{\varepsilon_{k}}-X_{s}\right\|_{H} v(d z) d s\right) \\
& \leq C \mathbb{E}\left(\int_{0}^{T} \int_{\mathbb{X}} L_{f}(s, z)\left(1+\left\|X_{s}^{\varepsilon_{k}}\right\|_{H}\right)\left|\varphi_{\varepsilon_{k}}(s, z)-1\right|\left\|X_{s}^{\varepsilon_{k}}-X_{s}\right\|_{H} \nu(d z) d s\right) \\
& \leq C \delta \mathbb{E}\left(\int_{A_{\delta, \varepsilon_{k}}^{c}} \int_{\mathbb{X}} L_{f}(s, z)\left(1+\left\|X_{s}^{\varepsilon_{k}}\right\|_{H}\right)\left|\varphi_{\varepsilon_{k}}(s, z)-1\right| v(d z) d s\right) \\
& +C \mathbb{E}\left(\int_{A_{\delta, \varepsilon_{k}}} \int_{\mathbb{X}} L_{f}(s, z)\left(1+\left\|X_{s}^{\varepsilon_{k}}\right\|_{H}\right)\left|\varphi_{\varepsilon_{k}}(s, z)-1\right|\left\|X_{s}^{\varepsilon_{k}}-X_{S}\right\|_{H} \mathcal{v}(d z) d s\right) \\
& \leq C \delta \mathbb{E}\left(\sup _{s \in[0, T]}\left(1+\left\|X_{s}^{\varepsilon_{k}}\right\|_{H}\right)\right) \sup _{\varphi \in S^{N}} \int_{0}^{T} \int_{\mathbb{X}} L_{f}(s, z)|\varphi(s, z)-1| v(d z) d s \\
& +C \mathbb{E}\left[\sup _{s \in[0, T]}\left(\left(1+\left\|X_{s}^{\varepsilon_{k}}\right\|_{H}\right)\left(\left\|X_{s}^{\varepsilon_{k}}-X_{s}\right\|_{H}\right)\right)\right. \\
& \left.\times \sup _{\varphi \in S^{N}} \int_{A_{\delta, \varepsilon_{k}}} \int_{\mathbb{X}} L_{f}(s, z)|\varphi(s, z)-1| v(d z) d s\right] \\
& \leq \delta C_{L_{f}, N}+C\left(\mathbb{E}\left(1+\sup _{s \in[0, T]}\left\|X_{S}^{\varepsilon_{k}}\right\|_{H}\right)^{4}\right)^{1 / 4}\left(\mathbb{E}\left(1+\sup _{s \in[0, T]}\left\|X_{s}^{\varepsilon_{k}}-X_{s}\right\|_{H}\right)^{4}\right)^{1 / 4} \\
& \cdot\left(\mathbb{E}\left(\sup _{\varphi \in S^{N}} \int_{A_{\delta, \varepsilon_{k}}} \int_{\mathbb{X}} L_{f}(s, z)|\varphi(s, z)-1| v(d z) d s\right)^{2}\right)^{1 / 2} .
\end{aligned}
$$

Similar as (5.14) and (5.15), we have

$$
\lim _{\varepsilon_{k} \rightarrow 0}\left|G\left(X^{\varepsilon_{k}}, \varphi_{\varepsilon_{k}}, X^{\varepsilon_{k}}\right)-G\left(X^{\varepsilon_{k}}, \varphi_{\varepsilon_{k}}, X\right)\right|=0 .
$$


On the other hand,

$$
\begin{aligned}
& \left|G\left(X^{\varepsilon_{k}}, \varphi_{\varepsilon_{k}}, X\right)-G\left(X, \varphi_{\varepsilon_{k}}, X\right)\right| \\
& \quad \leq C \mathbb{E}\left(\int_{0}^{T} \int_{\mathbb{X}}\left\|f\left(s, X_{s}^{\varepsilon_{k}}, z\right)-f\left(s, X_{s}, z\right)\right\|_{H}\left|\varphi_{\varepsilon_{k}}(s, z)-1\right|\left\|X_{S}\right\|_{H} v(d z) d s\right) \\
& \quad \leq C \mathbb{E}\left(\int_{0}^{T} \int_{\mathbb{X}} G_{f}(s, z)\left\|X_{s}^{\varepsilon_{k}}-X_{s}\right\|_{H}\left|\varphi_{\varepsilon_{k}}(s, z)-1\right|\left\|X_{S}\right\|_{H} v(d z) d s\right) .
\end{aligned}
$$

Using the similar arguments as proving (5.20), we have

$$
\lim _{\varepsilon_{k} \rightarrow 0}\left|G\left(X^{\varepsilon_{k}}, \varphi_{\varepsilon_{k}}, X\right)-G\left(X, \varphi_{\varepsilon_{k}}, X\right)\right|=0
$$

Combining (5.20), (5.21), and (5.18), we have (5.17).

\section{Lemma 5.4.}

$$
Y_{t}(\omega)=\mathcal{A}\left(t, X_{t}(\omega)\right) \quad \text { for } d t \times \overline{\mathbb{P}} \text {-almost all }(t, \omega)
$$

Proof. For $\phi \in \mathcal{K} \cap \mathcal{N} \cap L^{\infty}\left([0, T], L^{\beta+2}(\Omega, H)\right)$, applying the Itô's formula,

$$
\begin{aligned}
& e^{-\int_{0}^{t}\left(K_{s}+\rho\left(\phi_{s}\right)\right) d s}\left\|X_{t}^{\varepsilon_{k}}\right\|_{H}^{2}-\|x\|_{H}^{2} \\
& =\int_{0}^{t} e^{-\int_{0}^{s}\left(K_{l}+\rho\left(\phi_{l}\right)\right) d l}\left[-\left(K_{s}+\rho\left(\phi_{s}\right)\right)\left\|X_{s}^{\varepsilon_{k}}\right\|_{H}^{2}+2\left\langle\mathcal{A}\left(s, X_{s}^{\varepsilon_{k}}\right), X_{s}^{\varepsilon_{k}}\right\rangle_{V^{*}, V}\right. \\
& \left.\quad+2\left\langle\int_{\mathbb{X}} f\left(s, X_{s}^{\varepsilon_{k}}, z\right)\left(\varphi_{\varepsilon_{k}}(s, z)-1\right) v(d z), X_{s}^{\varepsilon_{k}}\right\rangle_{H, H}\right] d s \\
& \quad+\int_{0}^{t} e^{-\int_{0}^{s}\left(K_{l}+\rho\left(\phi_{l}\right)\right) d l} \int_{\mathbb{X}}\left[2 \varepsilon_{k}\left\langle f\left(s, X_{s-}^{\varepsilon_{k}}, z\right), X_{s-}^{\varepsilon_{k}}\right\rangle_{H, H}\right] \tilde{N}^{\varepsilon_{k}^{-1}} \varphi_{\varepsilon_{k}}(d s, d z) \\
& \quad+\int_{0}^{t} e^{-\int_{0}^{s}\left(K_{l}+\rho\left(\phi_{l}\right)\right) d l} \int_{\mathbb{X}}\left[\varepsilon_{k}^{2}\left\|f\left(s, X_{s-}^{\varepsilon_{k}}, z\right)\right\|_{H}^{2}\right] N^{\varepsilon_{k}^{-1} \varphi_{\varepsilon_{k}}}(d s, d z) .
\end{aligned}
$$

Notice that

$$
M_{\varepsilon_{k}}(t):=\int_{0}^{t} e^{-\int_{0}^{s}\left(K_{l}+\rho\left(\phi_{l}\right)\right) d l} \int_{\mathbb{X}}\left[2 \varepsilon_{k}\left\langle f\left(s, X_{s-}^{\varepsilon_{k}}, z\right), X_{s-}^{\varepsilon_{k}}\right\rangle_{H, H}\right] \tilde{N}^{\varepsilon_{k}^{-1} \varphi_{\varepsilon_{k}}}(d s, d z)
$$


is a square integrable martingale, we have

$$
\begin{aligned}
& \mathbb{E}\left(e^{-\int_{0}^{t}\left(K_{s}+\rho\left(\phi_{s}\right)\right) d s}\left\|X_{t}^{\varepsilon_{k}}\right\|_{H}^{2}\right)-\|x\|_{H}^{2} \\
& =-\mathbb{E}\left(\int _ { 0 } ^ { t } e ^ { - \int _ { 0 } ^ { s } ( K _ { l } + \rho ( \phi _ { l } ) ) d l } ( K _ { s } + \rho ( \phi _ { s } ) ) \left(\left\|X_{s}^{\varepsilon_{k}}-\phi_{s}\right\|_{H}^{2}\right.\right. \\
& \left.\left.+2\left\langle X_{s}^{\varepsilon_{k}}, \phi_{s}\right\rangle_{H, H}-\left\|\phi_{s}\right\|_{H}^{2}\right) d s\right) \\
& +\mathbb{E}\left(\int _ { 0 } ^ { t } e ^ { - \int _ { 0 } ^ { s } ( K _ { l } + \rho ( \phi _ { l } ) ) d l } \left(2\left\langle\mathcal{A}\left(s, X_{s}^{\varepsilon_{k}}\right)-\mathcal{A}\left(s, \phi_{s}\right), X_{s}^{\varepsilon_{k}}-\phi_{s}\right\rangle_{V^{*}, V}\right.\right. \\
& \left.\left.+2\left\langle\mathcal{A}\left(s, \phi_{s}\right), X_{s}^{\varepsilon_{k}}-\phi_{s}\right\rangle_{V^{*}, V}+2\left\langle\mathcal{A}\left(s, X_{s}^{\varepsilon_{k}}\right), \phi_{s}\right\rangle_{V^{*}, V}\right) d s\right) \\
& +\mathbb{E}\left(\int_{0}^{t} e^{-\int_{0}^{s}\left(K_{l}+\rho\left(\phi_{l}\right)\right) d l}\right. \\
& \left.\times\left(2\left\langle\int_{\mathbb{X}} f\left(s, X_{s}^{\varepsilon_{k}}, z\right)\left(\varphi_{\varepsilon_{k}}(s, z)-1\right) v(d z), X_{s}^{\varepsilon_{k}}\right\rangle_{H, H}\right) d s\right) \\
& +\mathbb{E}\left(\varepsilon_{k} \int_{0}^{t} e^{-\int_{0}^{s}\left(K_{l}+\rho\left(\phi_{l}\right)\right) d l} \int_{\mathbb{X}}\left\|f\left(s, X_{s}^{\varepsilon_{k}}, z\right)\right\|_{H}^{2} \varphi_{\varepsilon_{k}}(s, z) v(d z) d s\right) \\
& \leq-\mathbb{E}\left(\int_{0}^{t} e^{-\int_{0}^{s}\left(K_{l}+\rho\left(\phi_{l}\right)\right) d l}\left(K_{s}+\rho\left(\phi_{s}\right)\right)\left(2\left\langle X_{s}^{\varepsilon_{k}}, \phi_{s}\right\rangle_{H, H}-\left\|\phi_{s}\right\|_{H}^{2}\right) d s\right) \\
& +\mathbb{E}\left(\int _ { 0 } ^ { t } e ^ { - \int _ { 0 } ^ { s } ( K _ { l } + \rho ( \phi _ { l } ) ) d l } \left(2\left\langle\mathcal{A}\left(s, \phi_{s}\right), X_{s}^{\varepsilon_{k}}-\phi_{s}\right\rangle_{V^{*}, V}\right.\right. \\
& \left.\left.+2\left\langle\mathcal{A}\left(s, X_{s}^{\varepsilon_{k}}\right), \phi_{s}\right\rangle_{V^{*}, V}\right) d s\right) \\
& +\mathbb{E}\left(\int_{0}^{t} e^{-\int_{0}^{s}\left(K_{l}+\rho\left(\phi_{l}\right)\right) d l}\right. \\
& \left.\times\left(2\left\langle\int_{\mathbb{X}} f\left(s, X_{s}^{\varepsilon_{k}}, z\right)\left(\varphi_{\varepsilon_{k}}(s, z)-1\right) v(d z), X_{s}^{\varepsilon_{k}}\right\rangle_{H, H}\right) d s\right) \\
& +\mathbb{E}\left(\varepsilon_{k} \int_{0}^{t} e^{-\int_{0}^{s}\left(K_{l}+\rho\left(\phi_{l}\right)\right) d l} \int_{\mathbb{X}}\left\|f\left(s, X_{s}^{\varepsilon_{k}}, z\right)\right\|_{H}^{2} \varphi_{\varepsilon_{k}}(s, z) v(d z) d s\right) .
\end{aligned}
$$

By (i) of Lemma 5.1, we get

$$
\begin{aligned}
\mathbb{E} & {\left[\int_{0}^{T} \psi_{t}\left(e^{-\int_{0}^{t}\left(K_{s}+\rho\left(\phi_{s}\right)\right) d s}\left\|X_{t}\right\|_{H}^{2}-\|x\|_{H}^{2}\right) d t\right] } \\
& \leq \liminf _{\varepsilon_{k} \rightarrow 0} \mathbb{E}\left[\int_{0}^{T} \psi_{t}\left(e^{-\int_{0}^{t}\left(K_{s}+\rho\left(\phi_{s}\right)\right) d s}\left\|X_{t}^{\varepsilon_{k}}\right\|_{H}^{2}-\|x\|_{H}^{2}\right) d t\right] .
\end{aligned}
$$


By Lemma 4.1,

$$
\begin{aligned}
& \mathbb{E}\left(\varepsilon_{k} \int_{0}^{t} e^{-\int_{0}^{s}\left(K_{l}+\rho\left(\phi_{l}\right)\right) d l} \int_{\mathbb{X}}\left\|f\left(s, X_{s}^{\varepsilon_{k}}, z\right)\right\|_{H}^{2} \varphi_{\varepsilon_{k}}(s, z) v(d z) d s\right) \\
& \quad \leq \mathbb{E}\left(\varepsilon_{k} \int_{0}^{t} \int_{\mathbb{X}}\left(1+\left\|X_{s}^{\varepsilon_{k}}\right\|_{H}\right)^{2} L_{f}^{2}(s, z) \varphi_{\varepsilon_{k}}(s, z) v(d z) d s\right) \\
& \quad \leq \varepsilon_{k} \mathbb{E}\left(\left(1+\sup _{s \in[0, T]}\left\|X_{s}^{\varepsilon_{k}}\right\|_{H}\right)^{2}\right) \sup _{\varphi \in S^{N}} \int_{0}^{T} \int_{\mathbb{X}} L_{f}^{2}(s, z) \varphi(s, z) v(d z) d s \\
& \quad \leq \varepsilon_{k} C_{L_{f}, 2,2, N} .
\end{aligned}
$$

Combining from (5.22) to (5.24), and Lemma 5.3, we infer

$$
\begin{aligned}
& \mathbb{E}\left[\int_{0}^{T} \psi_{t}\left(e^{-\int_{0}^{t}\left(K_{s}+\rho\left(\phi_{s}\right)\right) d s}\left\|X_{t}\right\|_{H}^{2}-\|x\|_{H}^{2}\right) d t\right] \\
& \leq-\mathbb{E}\left[\int_{0}^{T} \psi_{t} \int_{0}^{t} e^{-\int_{0}^{s}\left(K_{l}+\rho\left(\phi_{l}\right)\right) d l}\left(K_{s}+\rho\left(\phi_{s}\right)\right)\left(2\left\langle X_{s}, \phi_{S}\right\rangle_{H, H}-\left\|\phi_{s}\right\|_{H}^{2}\right) d s d t\right] \\
& \quad+\mathbb{E}\left[\int _ { 0 } ^ { T } \psi _ { t } \int _ { 0 } ^ { t } e ^ { - \int _ { 0 } ^ { s } ( K _ { l } + \rho ( \phi _ { l } ) ) d l } \left(2\left\langle\mathcal{A}\left(s, \phi_{s}\right), X_{s}-\phi_{s}\right\rangle_{V^{*}, V}\right.\right. \\
& \left.\left.\quad+2\left\langle Y_{s}, \phi_{s}\right\rangle_{V^{*}, V}\right) d s d t\right] \\
& \quad+\mathbb{E}\left[\int_{0}^{T} \psi_{t} \int_{0}^{t} e^{-\int_{0}^{s}\left(K_{l}+\rho\left(\phi_{l}\right)\right) d l}\right. \\
& \left.\quad \times\left(2\left\langle\int_{\mathbb{X}} f\left(s, X_{s}, z\right)(\varphi(s, z)-1) v(d z), X_{S}\right\rangle_{H, H}\right) d s d t\right] .
\end{aligned}
$$

On the other hand, by (5.16), we have

$$
\begin{aligned}
& \mathbb{E}\left(e^{-\int_{0}^{t}\left(K_{s}+\rho\left(\phi_{s}\right)\right) d s}\left\|X_{t}\right\|_{H}^{2}-\|x\|_{H}^{2}\right) \\
&=- \mathbb{E}\left(\int_{0}^{t} e^{-\int_{0}^{s}\left(K_{l}+\rho\left(\phi_{l}\right)\right) d l}\left(K_{s}+\rho\left(\phi_{s}\right)\right)\left\|X_{S}\right\|_{H}^{2} d s\right) \\
&+\mathbb{E}\left(\int_{0}^{t} e^{-\int_{0}^{s}\left(K_{l}+\rho\left(\phi_{l}\right)\right) d l} 2\left\langle Y_{s}, X_{s}\right\rangle_{V^{*}, V} d s\right) \\
&+\mathbb{E}\left(\int_{0}^{t} e^{-\int_{0}^{s}\left(K_{l}+\rho\left(\phi_{l}\right)\right) d l}\left(2\left\langle\int_{\mathbb{X}} f\left(s, X_{s}, z\right)(\varphi(s, z)-1) v(d z), X_{s}\right\rangle_{H, H}\right) d s\right) .
\end{aligned}
$$


By (5.25) and (5.26), we have

$$
\begin{aligned}
& \mathbb{E}\left[\int _ { 0 } ^ { T } \psi _ { t } \int _ { 0 } ^ { t } e ^ { - \int _ { 0 } ^ { s } ( K _ { l } + \rho ( \phi _ { l } ) ) d l } \left(-\left(K_{s}+\rho\left(\phi_{s}\right)\right)\left\|X_{s}-\phi_{s}\right\|_{H}^{2}\right.\right. \\
& \left.\left.\quad+2\left\langle\mathcal{A}\left(s, \phi_{s}\right)-Y_{s}, X_{s}-\phi_{s}\right\rangle_{V^{*}, V}\right) d s d t\right] \leq 0 .
\end{aligned}
$$

Put $\phi=X-\eta \tilde{\phi} v$ for $\tilde{\phi} \in L^{\infty}([0, T] \times \Omega ; d t \times \overline{\mathbb{P}} ; \mathbb{R})$ and $v \in V$, divide both sides by $\eta$ and let $\eta \rightarrow 0$, then we have

$$
\mathbb{E}\left[\int_{0}^{T} \psi_{t} \int_{0}^{t} e^{-\int_{0}^{s}\left(K_{l}+\rho\left(\phi_{l}\right)\right) d l}\left(2 \tilde{\phi}_{s}\left\langle\mathcal{A}\left(s, \phi_{s}\right)-Y_{s}, v\right\rangle_{V^{*}, V}\right) d s d t\right] \leq 0 .
$$

Hence, $Y=\mathcal{A}(\cdot, X)$.

Proposition 5.1. $X(\omega)$ solves the following equation:

$$
X_{t}(\omega)=x+\int_{0}^{t} \mathcal{A}\left(s, X_{s}(\omega)\right) d s+\int_{0}^{t} \int_{\mathbb{X}} f\left(s, X_{s}(\omega), z\right)(\varphi(s, z)(\omega)-1) v(d z) d s,
$$

which has an unique solution in $C([0, T], H) \cap L^{\alpha}([0, T], V)$.

Proof. The equation (5.27) follows from Lemmas 5.1-5.4. The proof of the uniqueness is standard, and it is omitted.

Lemma 5.5. There exists a subsequence $\varpi_{k}$, such that

$$
\lim _{\varpi_{k} \rightarrow 0} \sup _{t \in[0, T]}\left\|X_{t}^{\varpi_{k}}-X_{t}\right\|_{H}^{2}=0, \quad \overline{\mathbb{P}} \text {-a.s. }
$$

\section{Proof.}

Set $L_{t}^{\varepsilon_{k}}=X_{t}^{\varepsilon_{k}}-X_{t}$. Then

$$
\begin{aligned}
& e^{-\int_{0}^{t}\left(K_{s}+\rho\left(X_{s}\right)\right) d s}\left\|L_{t}^{\varepsilon_{k}}\right\|_{H}^{2} \\
& =\int_{0}^{t} e^{-\int_{0}^{s}\left(K_{r}+\rho\left(X_{r}\right)\right) d r}\left(-\left(K_{S}+\rho\left(X_{S}\right)\right)\left\|L_{s}^{\varepsilon_{k}}\right\|_{H}^{2}\right. \\
& \left.\quad+2\left\langle\mathcal{A}\left(s, X_{s}^{\varepsilon_{k}}\right)-\mathcal{A}\left(s, X_{S}\right), L_{s}^{\varepsilon_{k}}\right\rangle_{V^{*}, V}\right) d s \\
& \quad+2 \int_{0}^{t} e^{-\int_{0}^{s}\left(K_{r}+\rho\left(X_{r}\right)\right) d r}\left\langle\int_{\mathbb{X}} f\left(s, X_{s}^{\varepsilon_{k}}, z\right)\left(\varphi_{\varepsilon_{k}}(s, z)-1\right) v(d z)\right. \\
& \left.\quad-\int_{\mathbb{X}} f\left(s, X_{s}, z\right)(\varphi(s, z)-1) v(d z), L_{s}^{\varepsilon_{k}}\right\rangle_{H, H} d s
\end{aligned}
$$




$$
\begin{aligned}
& +2 \varepsilon_{k} \int_{0}^{t} e^{-\int_{0}^{s}\left(K_{r}+\rho\left(X_{r}\right)\right) d r}\left\langle\int_{\mathbb{X}} f\left(s, X_{s}^{\varepsilon_{k}}, z\right), L_{s}^{\varepsilon_{k}}\right\rangle_{H, H} \tilde{N}^{\varepsilon_{k}^{-1} \varphi_{\varepsilon_{k}}}(d z, d s) \\
& +\varepsilon_{k}^{2} \int_{0}^{t} e^{-\int_{0}^{s}\left(K_{r}+\rho\left(X_{r}\right)\right) d r} \int_{\mathbb{X}}\left\|f\left(s, X_{s}^{\varepsilon_{k}}, z\right)\right\|_{H}^{2} N^{\varepsilon_{k}-1} \varphi_{\varepsilon_{k}}(d z, d s) \\
= & I_{1}(t)+I_{2}(t)+I_{3}(t)+I_{4}(t) .
\end{aligned}
$$

(H2) implies

$$
I_{1}(t) \leq 0 .
$$

By (5.19) and (5.20), we have

$$
\begin{aligned}
& \mathbb{E}\left(\sup _{t \in[0, T]}\left|\int_{0}^{t} e^{-\int_{0}^{s}\left(K_{r}+\rho\left(X_{r}\right)\right) d r}\left\langle\int_{\mathbb{X}} f\left(s, X_{s}^{\varepsilon_{k}}, z\right)\left(\varphi_{\varepsilon_{k}}(s, z)-1\right) v(d z), L_{s}^{\varepsilon_{k}}\right\rangle_{H, H} d s\right|\right) \\
& \quad \leq \mathbb{E}\left(\int_{0}^{T} \int_{\mathbb{X}}\left\|f\left(s, X_{s}^{\varepsilon_{k}}, z\right)\right\|_{H}\left|\varphi_{\varepsilon_{k}}(s, z)-1\right|\left\|L_{s}^{\varepsilon_{k}}\right\|_{H} v(d z) d s\right) \\
& \quad \leq \mathbb{E}\left(\int_{0}^{T} \int_{\mathbb{X}}\left\|X_{s}^{\varepsilon_{k}}\right\|_{H} L_{f}(s, z)\left|\varphi_{\varepsilon_{k}}(s, z)-1\right|\left\|L_{s}^{\varepsilon_{k}}\right\|_{H} v(d z) d s\right) \rightarrow 0, \quad \text { as } \varepsilon_{k} \rightarrow 0 .
\end{aligned}
$$

Then it is not difficulty to obtain

$$
\lim _{\varepsilon_{k} \rightarrow 0} \mathbb{E}\left(\sup _{t \in[0, T]}\left|I_{2}(t)\right|\right)=0 .
$$

For $I_{3}$,

$$
\begin{aligned}
& \mathbb{E}\left(\sup _{t \in[0, T]}\left|I_{3}(t)\right|\right) \\
& \leq \mathbb{E}\left(\int_{0}^{T} \int_{\mathbb{X}} 4 \varepsilon_{k}^{2}\left\|L_{s}^{\varepsilon_{k}}\right\|_{H}^{2}\left\|f\left(s, X_{s}^{\varepsilon_{k}}, z\right)\right\|_{H}^{2} N^{\varepsilon^{-1}} \varphi_{\varepsilon_{k}}(d s, d z)\right)^{1 / 2} \\
& \leq 2 \mathbb{E}\left(\sqrt{\varepsilon_{k}} \sup _{s \in[0, T]}\left\|L_{s}^{\varepsilon_{k}}\right\|_{H}\right. \\
&\left.\times\left(\int_{0}^{T} \int_{\mathbb{X}} \varepsilon_{k}\left\|f\left(s, X_{s}^{\varepsilon_{k}}, z\right)\right\|_{H}^{2} N^{\varepsilon_{k}-1} \varphi_{\varepsilon_{k}}(d s, d z)\right)^{1 / 2}\right) \\
& \leq 2 \sqrt{\varepsilon_{k}}\left(\mathbb{E}\left(\sup _{t \in[0, T]}\left\|L_{t}^{\varepsilon_{k}}\right\|_{H}^{2}\right)\right)^{1 / 2} \\
& \times\left(\mathbb{E}\left(\int_{0}^{T} \int_{\mathbb{X}}\left\|f\left(s, X_{s}^{\varepsilon_{k}}, z\right)\right\|_{H}^{2} \varphi_{\varepsilon_{k}}(s, z) v(d z) d s\right)\right)^{1 / 2} \\
& \leq 2 \sqrt{\varepsilon_{k}}\left(\mathbb{E}\left(\sup _{t \in[0, T]}\left\|L_{t}^{\varepsilon_{k}}\right\|_{H}^{2}\right)\right)^{1 / 2}
\end{aligned}
$$




$$
\begin{aligned}
& \times\left(\mathbb{E}\left(1+\sup _{t \in[0, T]}\left\|X_{t}^{\varepsilon_{k}}\right\|_{H}^{2}\right) \sup _{\varphi \in S^{N}} \int_{0}^{T} \int_{\mathbb{X}} L_{f}^{2}(s, z) \varphi(s, z) v(d z) d s\right)^{1 / 2} \\
\rightarrow & 0, \quad \text { as } \varepsilon_{k} \rightarrow 0 .
\end{aligned}
$$

For $I_{4}$

$$
\begin{aligned}
& \mathbb{E}\left(\sup _{t \in[0, T]}\left|I_{4}(t)\right|\right) \\
& \quad \leq \varepsilon_{k} \mathbb{E}\left(\int_{0}^{T} \int_{\mathbb{X}}\left\|f\left(s, X_{s}^{\varepsilon_{k}}, z\right)\right\|_{H}^{2} \varphi_{\varepsilon_{k}}(s, z) v(d z) d s\right) \\
& \quad \leq \varepsilon_{k} \mathbb{E}\left(1+\sup _{t \in[0, T]}\left\|X_{t}^{\varepsilon_{k}}\right\|_{H}^{2}\right) \sup _{\varphi \in S^{N}} \int_{0}^{T} \int_{\mathbb{X}} L_{f}^{2}(s, z) \varphi(s, z) v(d z) d s \\
& \quad \rightarrow 0, \quad \text { as } \varepsilon_{k} \rightarrow 0 .
\end{aligned}
$$

Combining (5.29)-(5.34), we have

$$
\lim _{\varepsilon_{k} \rightarrow 0} \mathbb{E}\left(\sup _{t \in[0, T]}\left(e^{-\int_{0}^{t}\left(K_{s}+\rho\left(X_{s}\right)\right) d s}\left\|L_{t}^{\varepsilon_{k}}\right\|_{H}^{2}\right)\right)=0 .
$$

Then

$$
\lim _{\varepsilon_{k} \rightarrow 0} \mathbb{E}\left(e^{-\int_{0}^{T}\left(K_{s}+\rho\left(X_{s}\right)\right) d s}\left(\sup _{t \in[0, T]}\left\|L_{t}^{\varepsilon_{k}}\right\|_{H}^{2}\right)\right)=0 .
$$

This implies that there exists a subsequence $\varpi_{k}$ such that $X^{\varpi_{k}}$ converges to $X \overline{\mathbb{P}}$-a.s.

\section{Verification of Condition 2.1}

Recall (4.5) and (3.1), we have

Theorem 6.1. Fixed $N \in \mathbb{N}$, and let $\varphi_{\varepsilon}, \varphi \in \tilde{\mathbb{A}}^{N}$ be such that $\varphi_{\varepsilon}$ converges in distribution to $\varphi$ as $\varepsilon \rightarrow 0$. Then

$$
\mathcal{G}^{\varepsilon}\left(\varepsilon N^{\varepsilon^{-1} \varphi_{\varepsilon}}\right) \Rightarrow \mathcal{G}^{0}\left(v_{T}^{\varphi}\right)
$$

Proof. Recall $\overline{\mathrm{M}}$ in Section 2 and notations in Proposition 4.1. Denote

$$
\Pi=\left(S^{N}, D\left([0, T], V^{*}\right), C\left([0, T], V^{*}\right), C\left([0, T], V^{*}\right), \bar{M}\right) .
$$

Proposition 4.1 implies that the laws of $\left\{\left(\varphi_{\varepsilon}, M^{\varepsilon}, Z^{\varepsilon}, Y^{\varepsilon}, \bar{N}\right), \varepsilon>0\right\}$ is tight in $\Pi$. Let $(\varphi, 0, Z, Y, \bar{N})$ be any limit point of the tight family. By the Skorohod's embedding theorem, taking a version on a new probability space if necessary, we may and will assume that

$$
\left(\varphi_{\varepsilon}, M^{\varepsilon}, Z^{\varepsilon}, Y^{\varepsilon}, \bar{N}\right) \longrightarrow(\varphi, 0, Z, Y, \bar{N}) \quad \text { in } \Pi, \overline{\mathbb{P}} \text {-a.s. }
$$


Set $\widetilde{X}^{\varepsilon}=x+M^{\varepsilon}+Z^{\varepsilon}+Y^{\varepsilon}$ and $X=x+Z+Y$. From the equation satisfied by the original processes, we may and will assume that $\tilde{X}$ still satisfy equation (4.4).

Using the fact that if $f_{n} \in D([0, T], \mathbb{R})$ and $\lim _{n \rightarrow \infty} f_{n}=0$ with the Skorokhod topology of $D([0, T], \mathbb{R})$, then $\lim _{n \rightarrow \infty} \sup _{t \in[0, T]}\left|f_{n}(t)\right|=0$. We have

$$
\lim _{\varepsilon \rightarrow 0} \sup _{t \in[0, T]}\left\|M^{\varepsilon}(t)\right\|_{V^{*}}=0, \quad \overline{\mathbb{P}} \text {-a.s. }
$$

Notice that

$$
\lim _{\varepsilon \rightarrow 0} \sup _{t \in[0, T]}\left\|Z^{\varepsilon}(t)-Z(t)\right\|_{V^{*}}=0, \quad \overline{\mathbb{P}} \text {-a.s. }
$$

and

$$
\lim _{\varepsilon \rightarrow 0} \sup _{t \in[0, T]}\left\|Y^{\varepsilon}(t)-Y(t)\right\|_{V^{*}}=0, \quad \overline{\mathbb{P}} \text {-a.s. }
$$

we have

$$
\lim _{\varepsilon \rightarrow 0} \sup _{t \in[0, T]}\left\|\tilde{X}^{\varepsilon}(t)-X(t)\right\|_{V^{*}}=0, \quad \overline{\mathbb{P}} \text {-a.s. }
$$

Finally, following the proof of Proposition 5.1 and Lemma 5.5, we can obtain $X$ is the unique solution of (5.27), and there exists a subsequence $\varpi_{k}$ that

$$
\lim _{\varpi_{k} \rightarrow 0} \sup _{t \in[0, T]}\left\|\tilde{X}^{\varpi_{k}}(t)-X(t)\right\|_{H}=0, \quad \overline{\mathbb{P}} \text {-a.s. }
$$

which implies this theorem.

We have finished to verify the second part of Condition 2.1. To obtain the first part of Condition 2.1, we just need to replace $\varepsilon \int_{\mathbb{X}} f\left(t, \widetilde{X}_{t-}^{\varepsilon}, z\right) \widetilde{N}^{\varepsilon^{-1}} \varphi_{\varepsilon}(d z, d t)$ by 0 in (4.4) and replacing $\varphi_{\varepsilon}$ by deterministic elements $g_{n}$ in in the proof of Lemma 4.4-Lemma 5.1, then we can similarly prove the following result.

Theorem 6.2. Recall $\mathcal{G}^{0}$ in (3.1). For all $N \in \mathbb{N}$, let $g_{n} \rightarrow g$ as $n \rightarrow \infty$. Then

$$
\lim _{n \rightarrow \infty} \sup _{t \in[0, T]}\left\|\mathcal{G}^{0}\left(v_{T}^{g_{n}}\right)(t)-\mathcal{G}^{0}\left(v_{T}^{g}\right)(t)\right\|_{H}=0 .
$$

\section{Acknowledgements}

We would like to thank an anonymous referee for his/her constructive suggestions which improved the presentation of the article. This work was started when the second author visited the first author at the Department of Mathematics, University of Macau. Jie Xiong's research is supported by Macao Science and Technology Fund FDCT 076/2012/A3 and Multi-Year Research Grants of the University of Macau Nos. MYRG2014-00015-FST and MYRG2014-00034-FST. Jianliang Zhai's research is supported by National Natural Science Foundation of China (NSFC) 
(No. 11431014, No. 11401557), the Fundamental Research Funds for the Central Universities (No. WK 3470000008), and Key Research Program of Frontier Sciences CAS (No. QYZDBSSW-SYS009).

\section{References}

[1] Applebaum, D. (2004). Lévy Processes and Stochastic Calculus. Cambridge Studies in Advanced Mathematics 93. Cambridge: Cambridge Univ. Press. MR2072890

[2] Bao, J. and Yuan, C. (2015). Large deviations for neutral functional SDEs with jumps. Stochastics 87 48-70. MR3306811

[3] Bessaih, H. and Millet, A. (2009). Large deviation principle and inviscid shell models. Electron. J. Probab. 14 2551-2579. MR2570011

[4] Brzeźniak, Z., Liu, W. and Zhu, J. (2014). Strong solutions for SPDE with locally monotone coefficients driven by Lévy noise. Nonlinear Anal. Real World Appl. 17 283-310. MR3158475

[5] Budhiraja, A., Chen, J. and Dupuis, P. (2013). Large deviations for stochastic partial differential equations driven by a Poisson random measure. Stochastic Process. Appl. 123 523-560. MR3003362

[6] Budhiraja, A. and Dupuis, P. (2000). A variational representation for positive functionals of infinite dimensional Brownian motion. Probab. Math. Statist. 20 39-61. MR1785237

[7] Budhiraja, A., Dupuis, P. and Maroulas, V. (2008). Large deviations for infinite dimensional stochastic dynamical systems. Ann. Probab. 36 1390-1420. MR2435853

[8] Budhiraja, A., Dupuis, P. and Maroulas, V. (2011). Variational representations for continuous time processes. Ann. Inst. Henri Poincaré Probab. Stat. 47 725-747. MR2841073

[9] Cardon-Weber, C. (1999). Large deviations for a Burgers'-type SPDE. Stochastic Process. Appl. 84 53-70. MR1720097

[10] Cerrai, S. and Röckner, M. (2004). Large deviations for stochastic reaction-diffusion systems with multiplicative noise and non-Lipschitz reaction term. Ann. Probab. 32 1100-1139. MR2044675

[11] Chenal, F. and Millet, A. (1997). Uniform large deviations for parabolic SPDEs and applications. Stochastic Process. Appl. 72 161-186. MR1486551

[12] Chow, P.L. (1992). Large deviation problem for some parabolic Itô equations. Comm. Pure Appl. Math. 45 97-120. MR1135925

[13] de Acosta, A. (1994). Large deviations for vector-valued Lévy processes. Stochastic Process. Appl. 51 75-115. MR1288284

[14] de Acosta, A. (2000). A general non-convex large deviation result with applications to stochastic equations. Probab. Theory Related Fields 118 483-521. MR1808373

[15] Duan, J. and Millet, A. (2009). Large deviations for the Boussinesq equations under random influences. Stochastic Process. Appl. 119 2052-2081. MR2519356

[16] Dupuis, P. and Ellis, R.S. (1997). A Weak Convergence Approach to the Theory of Large Deviations. Wiley Series in Probability and Statistics: Probability and Statistics. New York: Wiley. A WileyInterscience Publication. MR1431744

[17] Feng, J. and Kurtz, T.G. (2006). Large Deviations for Stochastic Processes. Mathematical Surveys and Monographs 131. Providence, RI: Amer. Math. Soc. MR2260560

[18] Ikeda, N. and Watanabe, S. (1981). Stochastic Differential Equations and Diffusion Processes. NorthHolland Mathematical Library 24. Amsterdam-New York: North-Holland; Tokyo: Kodansha, Ltd. MR0637061

[19] Kallianpur, G. and Xiong, J. (1996). Large deviations for a class of stochastic partial differential equations. Ann. Probab. 24 320-345. MR1387638 
[20] Liu, W. (2010). Large deviations for stochastic evolution equations with small multiplicative noise. Appl. Math. Optim. 61 27-56. MR2575313

[21] Liu, W. and Röckner, M. (2010). SPDE in Hilbert space with locally monotone coefficients. J. Funct. Anal. 259 2902-2922. MR2719279

[22] Manna, U., Sritharan, S.S. and Sundar, P. (2009). Large deviations for the stochastic shell model of turbulence. NoDEA Nonlinear Differential Equations Appl. 16 493-521. MR2525514

[23] Ren, J. and Zhang, X. (2008). Freidlin-Wentzell's large deviations for stochastic evolution equations. J. Funct. Anal. 254 3148-3172. MR2418622

[24] Röckner, M. and Zhang, T. (2007). Stochastic evolution equations of jump type: Existence, uniqueness and large deviation principles. Potential Anal. 26 255-279. MR2286037

[25] Röckner, M., Zhang, T. and Zhang, X. (2010). Large deviations for stochastic tamed 3D Navier-Stokes equations. Appl. Math. Optim. 61 267-285. MR2585144

[26] Sowers, R.B. (1992). Large deviations for a reaction-diffusion equation with non-Gaussian perturbations. Ann. Probab. 20 504-537. MR1143433

[27] Święch, A. and Zabczyk, J. (2011). Large deviations for stochastic PDE with Lévy noise. J. Funct. Anal. 260 674-723. MR2737394

[28] Xu, T. and Zhang, T. (2009). Large deviation principles for 2-D stochastic Navier-Stokes equations driven by Lévy processes. J. Funct. Anal. 257 1519-1545. MR2541279

[29] Yang, X., Zhai, J. and Zhang, T. (2015). Large deviations for SPDEs of jump type. Stoch. Dyn. 15 1550026,30 pp. MR3411897

[30] Zhai, J. and Zhang, T. (2015). Large deviations for 2-D stochastic Navier-Stokes equations driven by multiplicative Lévy noises. Bernoulli 21 2351-2392. MR3378470

[31] Zhang, T.S. (2000). On the small time asymptotics of diffusion processes on Hilbert spaces. Ann. Probab. 28 537-557. MR1782266

[32] Zhang, X. (2010). Stochastic Volterra equations in Banach spaces and stochastic partial differential equation. J. Funct. Anal. 258 1361-1425. MR2565842

Received August 2016 and revised February 2017 